\title{
THE CELLULAR GEOGRAPHY OF AURORA KINASES
}

\section{M ar Carmena and William C. Earnshaw}

Aurora is the name given to a family of highly conserved protein kinases with essential roles in many aspects of cell division. Yeasts have a single Aurora kinase, whereas mammals have three: Aurora A, B and C. During mitosis, Aurora kinases regulate the structure and function of the cytoskeleton and chromosomes and the interactions between these two at the kinetochore. They also regulate signalling by the spindle-assembly checkpoint pathway and cytokinesis. Perturbation of Aurora kinase expression or function might lead to cancer.

Aurora isthe name given to a family of serine/threonine protein kinases that regulate many processes during cell division. Aurora kinases are involved in the control of the CENTROSOME and nuclear cycles, and have essential functions in mitotic processes such as CHROM OSOME CONDENSATION, SPindle dynamiCS, KINETOCHORE-MICROTUBULE interactions, chromosomeorientation and establishment of the metaphase plate. They arealso required for the proper completion of cytokinesis.

The original aurora allelewas identified in a screen for D rosophila melanogaster mutants that were defective in spindle-pole behaviour, and was named after the aurora, a phenomenon of the night sky in the polar regions ${ }^{1}$. (Another kinaseidentified in the same screen was given the Spanish name for pole - Polo ${ }^{2}$.) Increase-in-ploidy 1 (Ipl1; REF. 3), thesingle essential Aurora kinase of Saccharomyces cerevisiae, was identified in a genetic screen for mutants that were defective in chromosome segregation. Schizosaccharomyces pombe al so has a singleAurora called Aurora-related kinase (Ark1; REFS4,5) which was isolated as a genethat, when present in a multicopy plasmid, suppressed the deleterious effects of overexpressing Xenopus Aurora A in S. pombe 4 . M etazoans have several Aurora kinases, with two paralogues known in D. melanogaster, Caenorhabditis elegans and Xenopus, and threein mammals. Because the kinases wereidentified independently many times, the nomenclature becameunwieldy, but it has now been simplified with the agreement to designate the family members Aurora A, B, and C6,7. The original D rosophila Aurora is now classified as Aurora A. A phylogenetic analysis showing the relationships between the kinase domains of various Aurora kinases is shown in FIG. 1. This diagram shows that the subfamilies $A$ and $B$ are very well defined in vertebrates, but the differences are not so obvious in invertebrates.

The three mammalian Aurora paralogues are very similar in sequence, in particular within the carboxyterminal catalytic domain, in which human Aurora A and $B$ share $71 \%$ identity. However, thethreeAuroras differ in the length and sequence of the amino-terminal domain ${ }^{8,9}$. The structure of the catalytic domain of human Aurora A was recently solved ${ }^{10,11}$. The high degree of sequence similarity between human Aurora $A$ and $B$ in this region is highlighted in FIG. 2, in which sequence conservation has been mapped on the surface of theAurora-A catalytic domain. This high degree of conservation must betaken into account when considering the specificity of Aurora kinase substrates and inhibitors (see below).

Surprisingly, given this level of similarity, the three mammalian Aurora kinases have very distinct localizations and functions (FIG. 3). Aurora-A kinases are associated with the centrosomefrom the time of centrosome duplication through to mitotic exit, and arealso associated with regions of microtubules proximal to centrosomes in mitosis. Aurora-B kinases form a complex with two other proteins, inner centromere protein (INCENP) and survivin, and behave as chromosomal 'passenger' proteins ${ }^{12}$. Passenger proteins associate with centromeric 


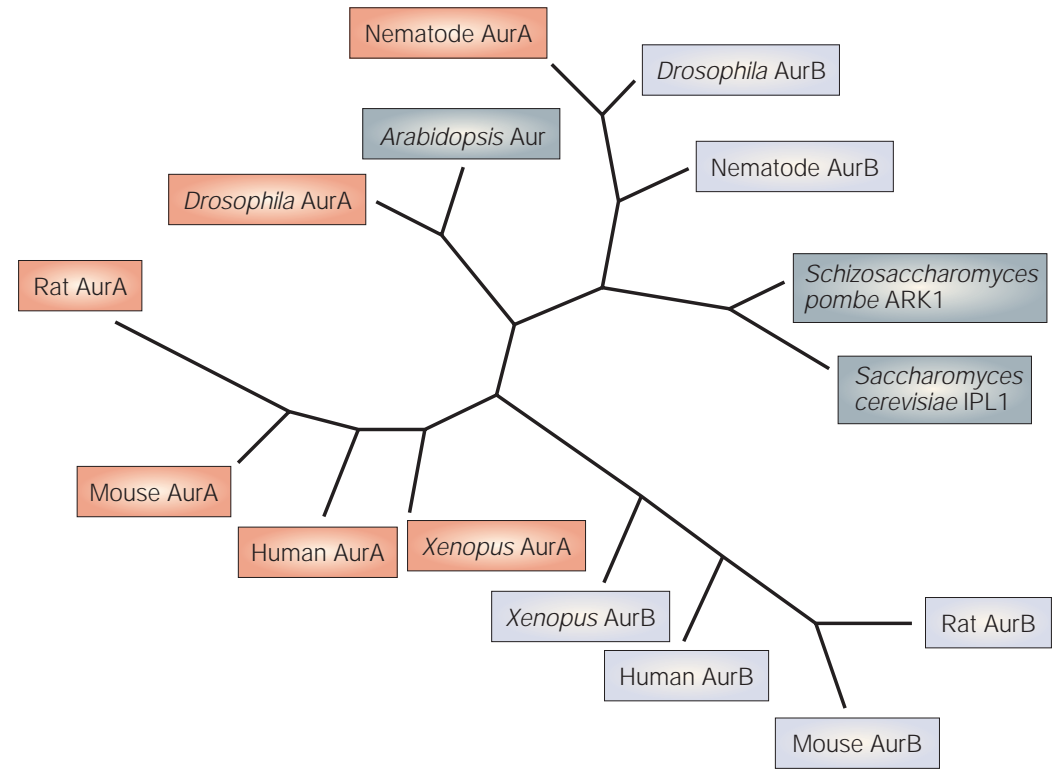

Figure 1 | The relationships between the kinase domains of the Aurora kinases. The vertebrate A- and B-type Aurora kinases (Aur) are readily distinguished, but the relationships between the invertebrate and plant enzymes are less clear-cut. The phylogenetic tree was calculated by maximum parsimony. The confidence estimate of the topology assignment was obtained by bootstrapping (randomized substitutions).

MICROTUBULE

A hollow tube, $25 \mathrm{~nm}$ in diameter, that is formed by the lateral association of 13 protofilaments, which are themselves polymers of $\alpha$ - and $\beta$-tubulin subunits.

\section{PARALOGUES}

Genes with related structureand function within the same species.

HETEROCHROM ATIN

A condensed form of chromatin containing few expressed genes and often rich in repeated DNA elements. It is commonly, but not exclusively, found around the centromere.

\section{MIDBODY}

A densebundle of microtubules embedded in an electron-dense matrix. Thisis derived from the central spindle during late telophaseand is localized within theintercellular bridge during cytokinesis.

SPINDLE MIDZONE Organized bundles of antiparallel microtubules that form during anaphaseand telophase. Signals from the central spindlearethought to be important for signalling the location of the cleavagefurrow.
HETEROCHROM ATIN early in mitosis (see BOX 1), transfer to thecentral spindle in anaphaseand are amongst thefirst proteins to localize at the cell cortex wherethe contractile ring subsequently forms. Chromosomal passenger proteins remain associated with the м IDвоDY during cytokinesis. The dramatic movements of passenger proteins during mitosis led to the proposal that they might have a role in the coordination of chromosomal and cytoskeletal events during the cell cycle ${ }^{12}$. Less is known about Aurora- $C$ kinases, which are specifically expressed at high levels in the testis and show centrosomal localization from anaphase to telophase ${ }^{13-16}$. One challenge for the future will be to dissect out the features that definethe substrate specificity, regulation and localization of each of theAurora kinases.

The expression level sof human Auroras and some of their associated polypeptides are elevated in certain types of cancer, and overexpression of Aurora A can induce transformation. These observations have lent new interest to thisfamily of kinases as potential drug targets for the development of new anti-cancer therapies.

\section{Aurora A: the polar aurora}

The defining characteristic of the Aurora-A subfamily has been its association with centrosomes and regions of microtubules that are proximal to the centrosome $\mathrm{e}^{1,17-19}$. Aurora A associates with the centrosomes that are separating during lateS/ early G2. This localization is dynamic and theprotein exchanges continuously with a cytoplasmic pool (FIG. 3; REF. 20). Low levels of Aurora A havealso been reported on the SPINDLE MIDZONE and midbody latein mitosis ${ }^{19,21,22}$. The association with the centrosome is directed independently both by the amino-terminal region and the carboxy-terminal catalytic domain, but does not require kinase activity $^{23,24}$. TPX2 (targeting protein for XKLP2), which has been implicated in Aurora-A activation (see below), is required for the localization of the kinaseto spindle microtubules, but not to SPINDLE POLES ${ }^{25}$.

Aurora A in centrosomeseparation and maturation. The high frequen cy of monopolar MITOTIC FIGURES in certain Drosophila aurora mutants indicated a potential rolefor the kinase in centrosome separation ${ }^{1}$. This was supported by ultrastructural analysis of D rosophila cells after they were treated with double-stranded Aurora-A DNA for RNA INTERFERENCE (RNAi). This showed thepresence of spindles with no cENTRIOLEs at onepoleand several centrioles at the other ${ }^{24}$. By contrast, centrosome duplication and separation seemed normal in one study using Aurora-A RNAi in C. elegan $s^{17}$. H owever, a second live-cell analysis of Aurora-A RNAi treatment in C. elegans showed that centrosomes first separate and then collapse back together ${ }^{26}$.

In order to become fully functional after they duplicateand separate, centrosomes must recruit a number of different proteins in a process known as maturation. In the absence of Aurora A, recruitment of several components of the PERICENTRIOLAR MATERIAL - including $\gamma$-TUBULIN - to the centrosomeis deficient, and the microtubule mass of spindles is decreased by about $60 \%$ (REFS 20,26). Furthermore, themorphology of the astral microtubule array is also aberrant. This might partly reflect an impaired function of factors that regulate microtubule dynamics, such as D rosophila transforming-acidiccoiled-coil protein (D-TACC; this seems to bethe orthologue of TACC3 in humans), and/or Eg5, a kinesin-like protein that is involved in spindle assembly. Both of these proteins are substrates of Aurora A in vitro 24,27.

Spindle assembly. A recent report has shown a link between Ran-GTP and AuroraA in spindleassembly ${ }^{28}$. Ran-GTP is a small GTPasethat is required for the polarity of nuclear transport, nuclear-envelope assembly and mitotic-spindle assembly in Xenopus extracts ${ }^{29-31}$. In mitosis, Ran-GTP releasesTPX 2 (and other centrosomeassociated proteins such as nuclear-mitotic-apparatus protein (N UMA)) from IM PORTIN- $\alpha$ and importin- $\beta$ (FIG. 4). The liberated TPX2 then binds Aurora A at the centrosomeand targetsit to themicrotubules proximal to the pole ${ }^{25}$. TPX2 also regulates the kinase activity of Aurora A, both by counteracting the activity of the protein phosphatase PP1 and stimulating Aurora-A autophosphorylation at Thr295 - a residue in the ACTIVATION LOOP of Aurora A that is essential for kinase activity ${ }^{28,32}$. Whether or not this activation requires microtubules is presently debated. So a Ran-GTP gradient is converted into a gradient of activeAurora $A$, and this isimportant for spindleassembly in vitro.

Meiotic maturation and metaphase I spindle orientation. Xenopus oocytes are arrested in prophase of the first meiotic division. Progesterone induces the activation of M-phase promoting factor (MPF; cyclindependent-kinase-1-cyclin-B-CKS1) and extracellular 
SPINDLE POLE

Theregion at theend of the mitotic spindle whereminus ends of themicrotubules are clustered together as a result of the action of various microtubulemotor proteins. In most animal cells, the spindle poleis centered around a centrosome. Plant spindles in somatic cells, however, lack focused centrosomes.

MITOTIC FIGURES A term used by cytologists to describemitotic cells visualized under themicroscope.

RNA INTERFERENCE (RNAi). A form of posttranscriptional genesilencing in which expression or transfection of double-stranded RNA induces degradation - by nucleases - of thehomologous endogenoustranscripts mimicking the effect of the reduction, or loss, of gene activity.

\section{CENTRIOLE}

A cylindrical array of 9 bundles of microtubules (usually triplets in animal cells) with other specialized appendages. Two centrioles, referred to as mother and daughter, arefound in the centre of centrosomesin animal cells.

PERICENTRIOLAR MATERIAL Region of the cytoplasm surrounding the two centrioles in the centrosome. Thisisthe region wheremicrotubule assembly is initiated by $\gamma$-tubulin.

$\gamma$-TUBULIN

A specialized isoform of tubulin that, together with several associated proteins, forms a ring-like complex that directs theinitiation of microtubule assembly.

IMPORTIN

A class of protein discovered because of the ability of the members to act as adaptors and carriers during theimport (and export) of proteins across the nuclear envelope. Importins are now known to have a second rolein regulating the assembly of themitotic spindle. signal-regulated kinase(ERK)/mitogen-activated protein kinase (MAPK), which transform the oocyteinto a mature egg. Aurora A was identified as a component of the progesterone-induced signalling pathway in a screen for proteins that weremodified in response to progesterone stimulation ${ }^{33}$. Aurora-A activation is an early event after progesterone activation, and its activity resultsin ERK/M APK signalling.

TheERK/M APK cascadeis initiated by translational upregulation of M OS, a M APK kinase kinase. Translation of M os messenger RNA requires polyadenylation and is dependent on cytoplasmic-polyadenylation-element binding protein (CPEB), a protein that binds within $3^{\prime}$ untranslated sequences. AuroraA is responsiblefor the early phosphorylation of CPEB and consequent upregulation of $\mathrm{M} \mathrm{OS}^{34}$. A later wave of CPEB phosphorylation depends on M PF. Xenopus Aurora A is also required downstream of $M P F$ for extrusion of the first POLAR BODY, meiotic-spindle positioning and exit from metaphasel (REFS 35,36). (Aurora B is required for polarbody extrusion in C. elegans ${ }^{37}$.) The Eg5 kinesin (an Aurora-A substrate) is involved in theAurora-mediated exit from metaphasel by an as-yet-unknown mechanism ${ }^{38}$. Aurora-A phosphorylation of CPEB also has an essential role in cell-cycle regulation in Xenopus early embryos, by upregulating thetranslation of cyclin B1.

Regulation of Aurora A activity. Regulation of AuroraA is complex and involves both phosphorylation/dephosphorylation and degradation. Phosphorylation stimulates kinase activity. Three phosphorylation sites have been identified in XenopusAurora A by mass spectrometry ${ }^{39}$. Phosphorylation of Thr295 (Thr288in human Aurora A) in theactivation loop is essential for kinase activity ${ }^{40}$. This residueis in a protein kinaseA (PKA) consensus motif, and PKA can phosphorylateand activate AuroraA in vitro ${ }^{40}$. However, this site also fits the consensus phosphorylation sequence that has been defined for S. cerevisiaelpl1 ((R/K)X(T/S) (I/L/V); REF. 41) and the equivalent residue (Thr260) in yeast Aurora has indeed been identified as an autophosphorylation site ${ }^{41}$. Ser 53 in the amino-terminal A Box is phosphorylated during $M$ phase and might have a role in the regulation of Aurora-A degradation (see below). Although thethird phosphorylation site to beidentified - Ser349 - is not essential for catalytic activity, S349D mutants block kinase activation, which indicates a possible structural or regulatory rolefor this modification ${ }^{39}$.

The phosphatase PP1 negatively regulates the Aurora kinases. The counteracting effect of PP1, which was first described for yeast I pl1 (REF. 42), has also been shown in Xenopus and human cell lines ${ }^{33,40,43,44}$. The activation of AuroraA by TPX 2 is at least partly due to antagonism of $P P 1^{28,32}$.

Aurora A is degraded in latemitosis/early G1 by the Cdh1/Fizzy-related form of theanaphase promoting complex/cyclosome (APC/C; the APC/C with Cdh1/Fizzy-related as a substrate recognition subunit $\left.{ }^{45,46}\right)$. Aurora A has a silent carboxy-terminal D-box (destruction box; FIG. 2), which is also present in Aurora $B^{47,48}$, but which is only functional in the presence of an

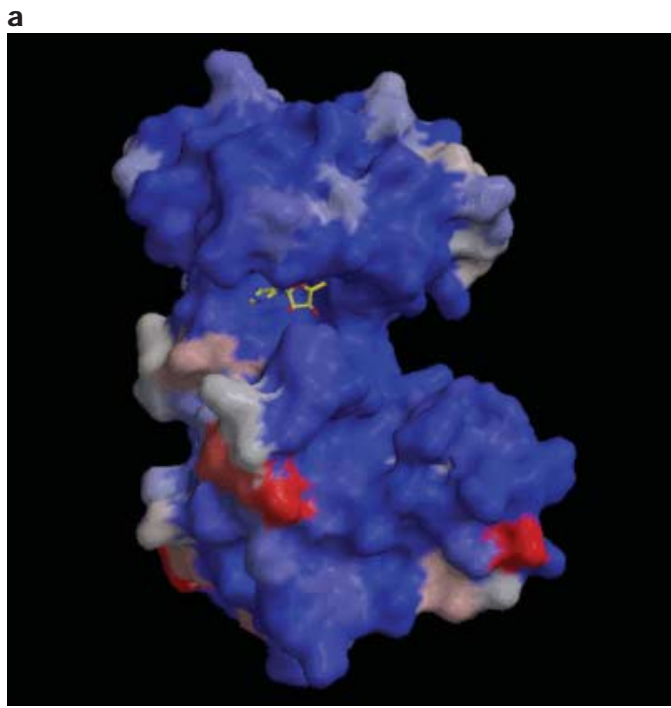

b
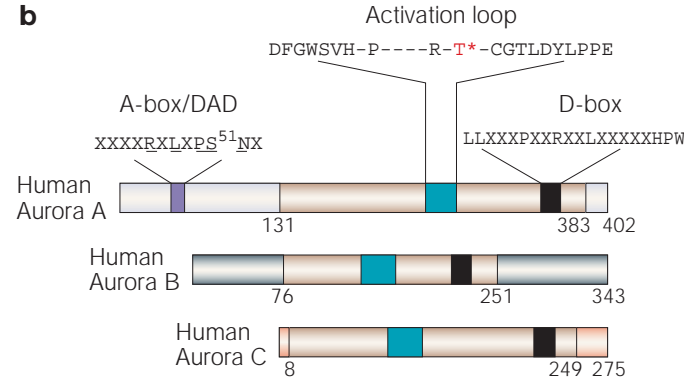

$\square \begin{aligned} & \text { Catalytic } \\ & \text { domain }\end{aligned} \begin{aligned} & \text { Activation } \\ & \text { loop }\end{aligned} \quad \begin{aligned} & \text { Destruction } \\ & \text { box }\end{aligned}$

Figure 2 | Structure of the Aurora kinases. a | The region of Aurora A and B surrounding the catalytic cleft is remarkably conserved. The figure shows the crystal structure of the kinase domain of Aurora A. The GRASP programme was used to obtain the surface representation of Aurora A. Surface mapping of amino-acid conservation was obtained with the Cosurf programme. A linear colour ramp represents the average conservation of each amino acid, ranging from 0.5 (red, divergent) to 1.0 (blue, conserved). b | The organization of human Aurora- $A,-B$ and $-C$ kinases. The position of the A-box/D-box-activating domain (DAD) and the D-box is shown, as is the position of the activation loop. These features have been characterized most thoroughly in Aurora A, and the boxes shown for Aurora B and $\mathrm{C}$ are approximations only.

amino-terminal A-box (also called the D-box-activating-domain (DAD) $)^{47}$. TheA-box/DAD is absent from Aurora $B$ and $C$, and their $D$-boxes are not targeted by the APC/C during mitotic exit. Phosphorylation of the A-box seems to make the Aurora A resistant to APC/Cmediated degradation. Interestingly, the D-box is recognized by both the Cdh1/Fizzy-related and Cdc20/Fizzy forms of the APC/C, but Aurora A is only targeted by theformer.

The recently identified Aurora-A-kinase-interacting protein (AIP), a negative regulator of Aurora $A$, is a conserved nuclear protein that interacts with the 


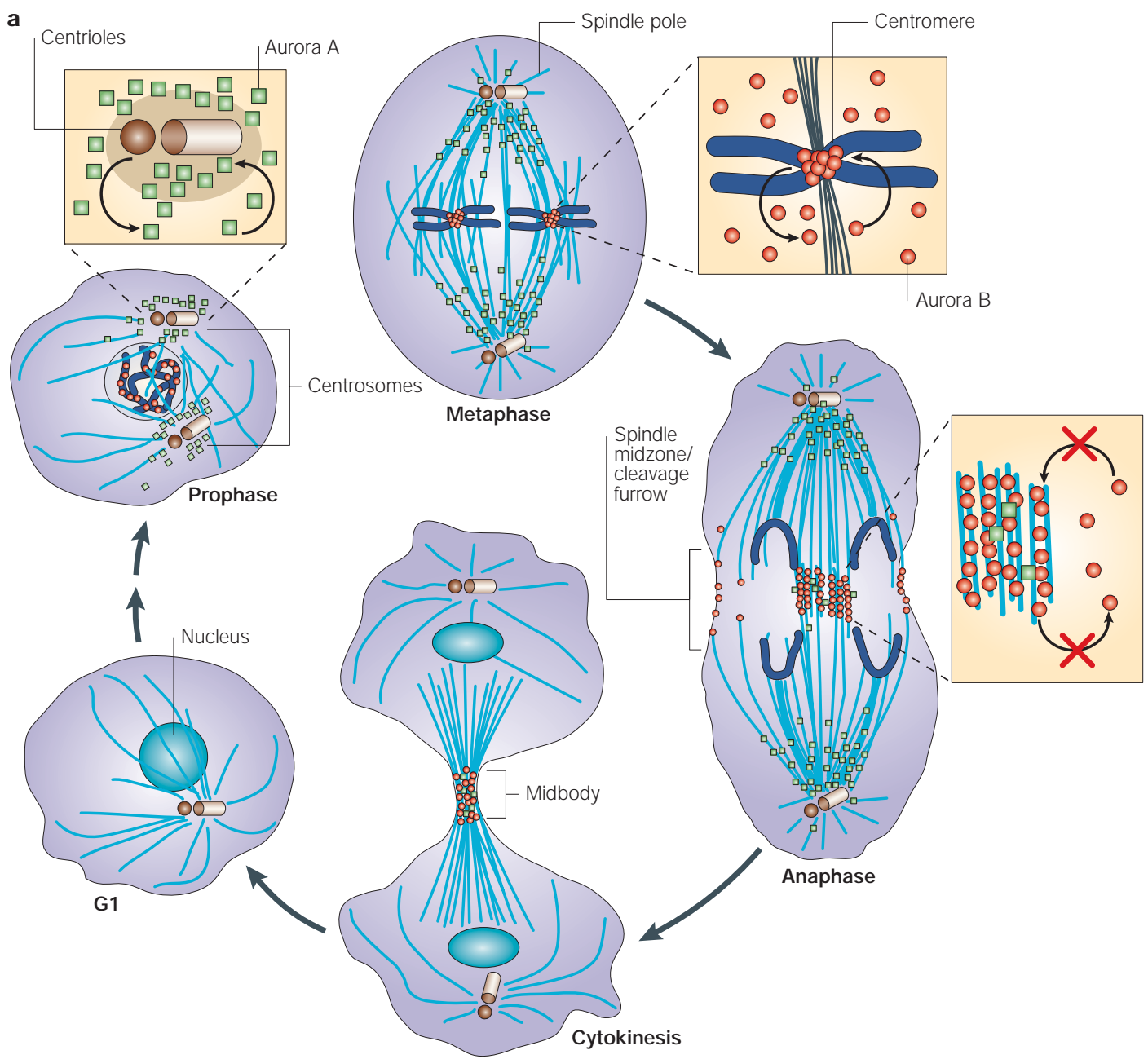

\section{ACTIVATION LOOP}

A conserved structural motif in kinase domains, which needs to be phosphorylated for full activation of the kinase

MPF

(M aturation-promoting factor/M - phase promoting factor). The complex of a B-type cyclin Cks1, and cyclindependent kinase 1, which is also referred to as $\mathrm{Cdc} 2$ or $\mathrm{p} 34$ depending on the species. This is themain enzymethat is responsiblefor entryinto $M$ phasein both meiosis and mitosis.

\section{POLAR BODY}

Either of thetwo small cells, each being formed during the successive divisions of meiosis, that forms as a result of division of a primary oocyte during its development to a mature ovum. The cytoplasm divides unequally during each division - the polar body is much smaller than the developing oocyte. Polar bodies eventually degenerate.

\section{b Metaphase}

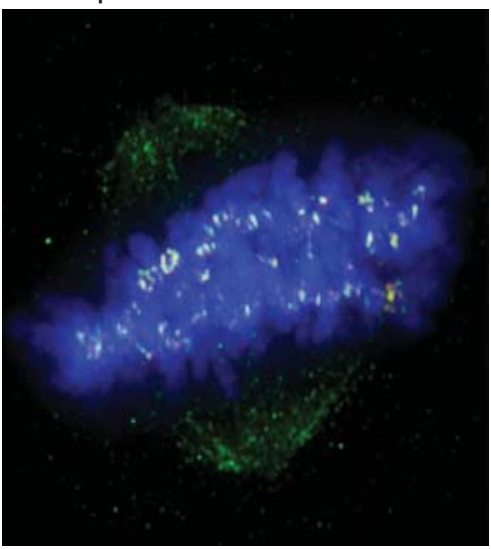

C Anaphase

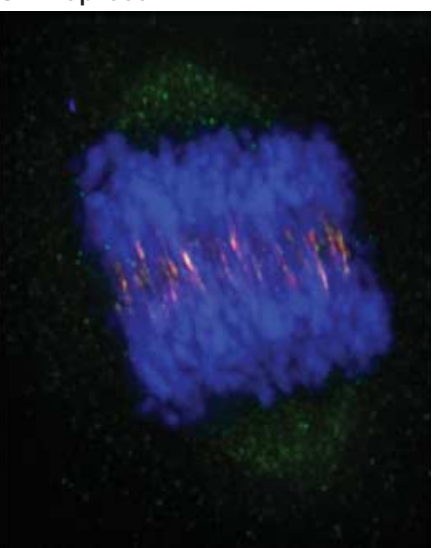

d Cytokinesis

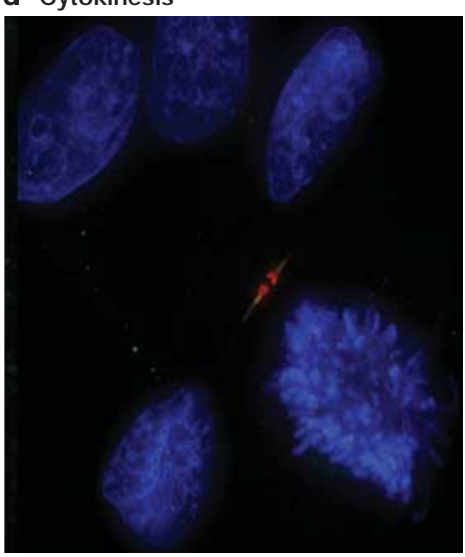

Figure 3 | Localization of Aurora kinases. a | The relative localization of Aurora A and Aurora B in mitotic cells is shown. The level of both kinases is substantially reduced in $\mathrm{G} 1$ cells. By prophase, Aurora A (green boxes) is concentrated around the centrosomes, whereas Aurora B (red circles) is nuclear. In metaphase, Aurora A is on the microtubules near the spindle poles, whereas Aurora B is located in the inner centromere. In anaphase, most Aurora $A$ is on the polar microtubules, but some might also be located in the spindle midzone. Aurora B is concentrated in the spindle midzone and at the cell cortex at the site of cleavage-furrow ingression. In cytokinesis, both kinases are concentrated in the midbody. The inset boxes show the results of fluorescence recovery after photobleaching (FRAP) studies, and indicate that Aurora A and B are dynamic at centrosomes and centromeres, respectively, but that Aurora B becomes immobile when targeted to the spindle midzone. Restoration microscopy of Aurora B (red staining), inner centromere protein (INCENP; green staining), and DNA (blue staining) in mitotic HeLa cells in (b) metaphase, (c) anaphase and (d) cytokinesis. Some background staining of INCENP on the spindle is seen in this experiment. 


\section{Box 1 A brief primer of mitosis}
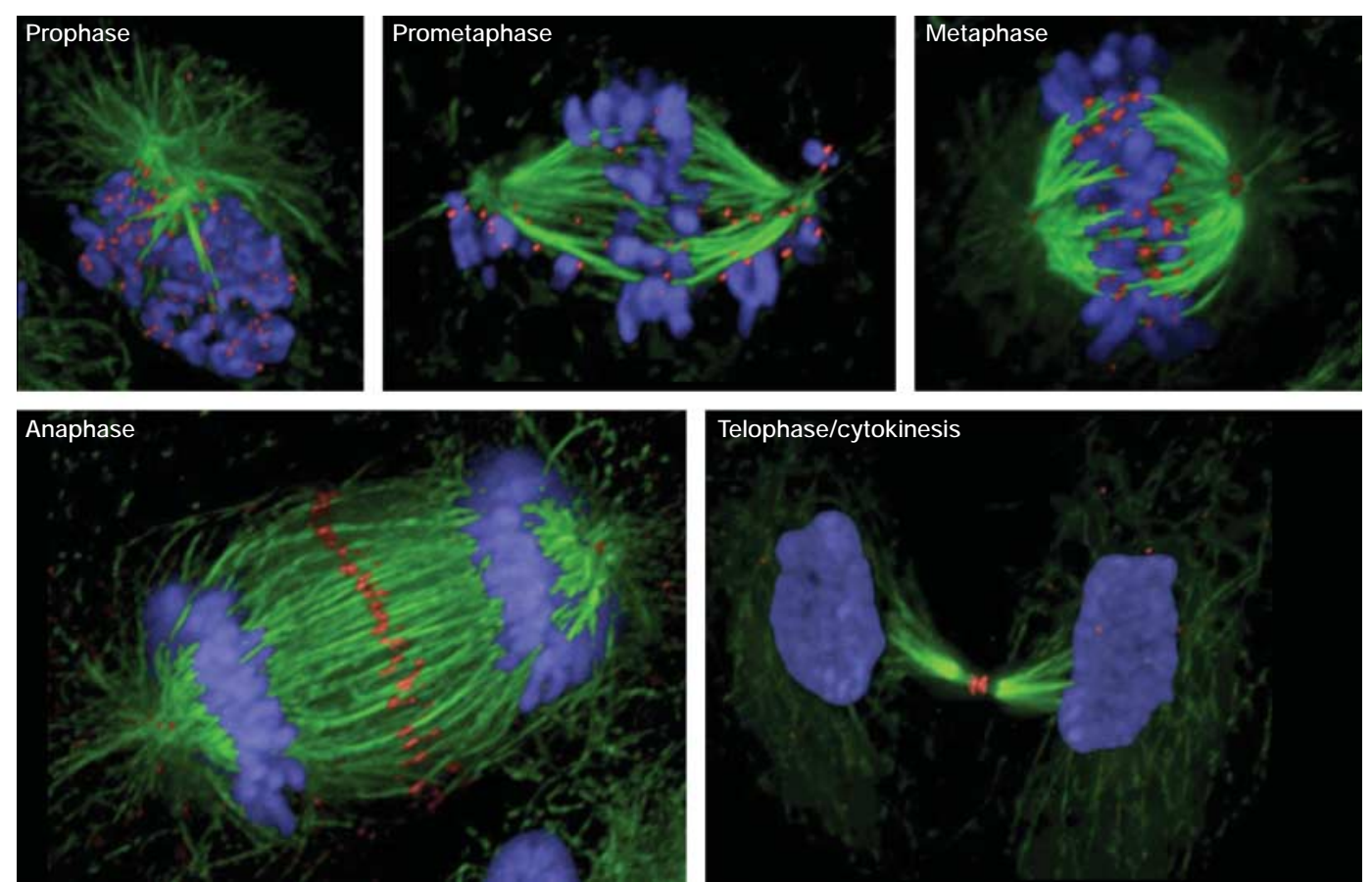

Mitosis begins with prophase (seefigure), as chromosomes condense within the intact nuclear envelope and the replicated centrosomes begin to separate. The centrosomes undergo a processtermed maturation, and begin to nucleate two asters of dynamic microtubules. Prometaphase (seefigure) begins with nuclear-envelope breakdown, which allows the kinetochores to bind to microtubules. Chromosomes that have successfully attached to microtubules emanating from both poles of the spindle move to the spindlemidzone, forming a highly dynamic METAPHASE PLATE. D uring metaphase (see figure), all chromosomes have achieved an alignment at the spindle midzone. The degradation of key mitotic regulators begins, culminating in the activation of the anaphase-promoting complex/cyclosome (APC/C). Action of the APC/C ultimately leads to activation of a protease, known as separase, that cleaves a key regulator of sister-chromatid pairing. This triggers the onset of anaphase - the coordinated separation of sister chromatids to opposite poles of the spindle (seefigure). D uring anaphase, the spindle midzone gradually becomes organized into bundles of microtubules that will ultimately have an important role in specifying the formation of the cleavage furrow. D uring telophase, the nuclear envelope reforms on the surface of the separated masses of sister chromatids, and during cytokinesis (see figure), the cortex constricts under the action of an actomyosin ring. Ultimately, the remnant of the spindle is left as a bundle of microtubules passing through an intercellular bridge and embedded in a dense matrix at the midbody. The figures show chromosomes (blue), microtubules (green), and centromere protein E (CENP-E; red) - a marker for kinetochores, the central spindle, and midbody.

Thedynamic group of chromosomes positioned roughly midway between spindlepoles prior to theonset of anaphase. These

chromosomes haveformed proper bipolar attachments (that is, their sister chromatids are connected to oppositespindle poles)

\section{PROTEASOME}

Protein complex responsiblefor degrading intracellular proteins that have been tagged for destruction by theaddition of ubiquitin.

ASTRAL MICROTUBULES Microtubules that extend in a radial array outwards from the spindle poles, excluding those microtubules that attach to kinetochores or form part of the organized spindlemidzone. kinase in vivo ${ }^{49}$. AIP was isolated as a dosage dependent suppressor of Aurora A that was ectopically overexpressed in budding yeast. In mammalian cells, AIP might downregulate Aurora through PROTEASOM E-dependent degradation ${ }^{49}$. Its normal function is not known.

\section{Aurora B: the equatorial Aurora}

Human Aurora B was first identified in a polymerase chain reaction screen for kinases that were overexpressed in tumours ${ }^{8}$. Rat Aurora B was identified in a screen to find kinases that affected S. cerevisiae proliferation when they were overexpressed ${ }^{50}$. Aurora-B kinases are chromosomal passenger proteins that are essential for a number of processes during mitosis. Aurora-B expression and activity in proliferating tissues are cell-cycle regulated: expression peaks at the G2-M transition, and kinase activity is maximal during mitosis $5^{8,50}$.
Thechromosomal-passenger local ization of Aurora B was originally shown by indirect immunofluorescence in mammalian cells, C. elegans and Drosophila ${ }^{37,50,51}$. A more recent analysis of the distribution of exogenous green fluorescent protein (GFP)-tagged Aurora B in living mammalian cells showed that the association of the kinase with centromeres during metaphase is dynamic: the protein exchanges continuously with the surrounding cytoplasmic pool ${ }^{52}$. Once the kinase associates with central spindle microtubules during anaphase (which requires kinase activity), its mobility is highly reduced. A subpopulation of the tagged Aurora al so seems to be transported by ASTRAL MICROTUBULES to the equatorial cell cortex ${ }^{52}$

Aurora B in chromosomebiorientation. After nuclearenvelope breakdown, prometaphase chromosomes rapidly establish attachments to a nearby spindle pole, 


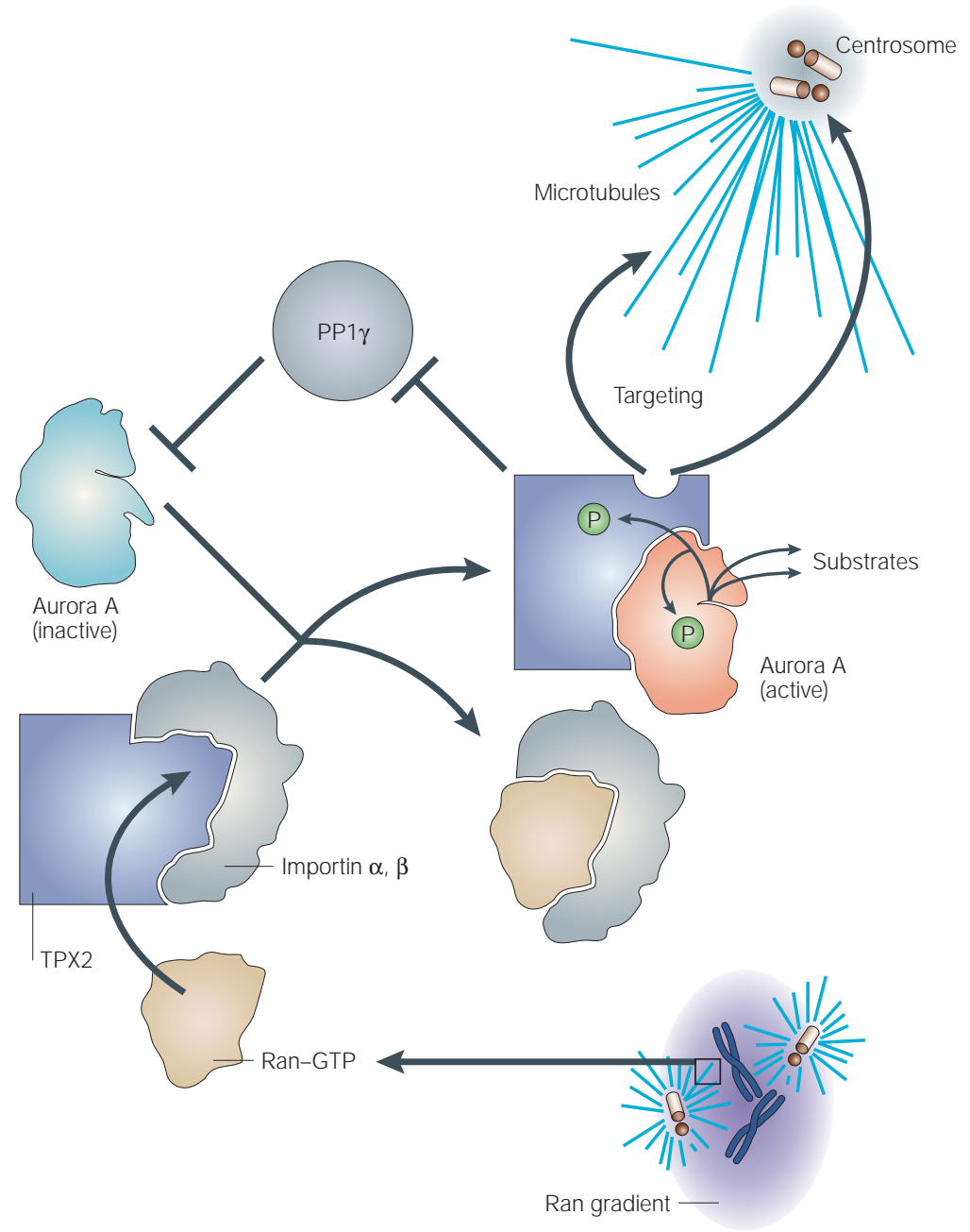

Figure 4 | Regulation of Aurora-A activity by Ran-GTP and TPX2. As cells enter mitosis, targeting protein for XKLP2 (TPX2) is in a complex with importins $\alpha$ or $\beta$. A gradient of Ran-GTP surrounding chromosomes (lower right) promotes the release of TPX2 from the importin. TPX2 then binds to Aurora A, which has been kept in an inactive state by protein phosphatase $1 \gamma$ (PP1 1). TPX2 interferes with PP1 action, enabling the kinase to autophosphorylate and activate itself and other substrates, including TPX2. TPX2 then also targets the kinase to microtubules proximal to the centrosome. Note that the kinase might not require continued association with TPX2 to phosphorylate other substrates.

SISTER CHROM ATIDS

Chromosomes that have been duplicated during $S$ phase. Sister chromatids are held together by cohesins until metaphase.

\section{CONGRESSION}

Themovement of correctly attached mitotic chromosomes to the metaphase plate at the midzone of the mitotic spindle. creating a 'monotelic' attachment (one sister kinetochore attached, the other one free; see FIG. 5). Eventually, the free kinetochore captures a microtubule from the opposite pole, yielding a bipolar (amphitelic) attachment that ultimately results in the positioning of the chromosome midway between the spindle poles at a metaphase plate. If both sister kinetochores attach to the same pole (syntelic attachment) the chromosome is unable to congress to the metaphase plate. Classic studies of meiotic cells by Nicklas and co-workers showed that tension within the spindle stabilizes amphitelic attachment ${ }^{53}$, and seems to correlate with silencing of a phospho-epitope on one or morekinetochore proteins that is recognized by the $3 \mathrm{~F} 3 / 2$ monoclonal antibody ${ }^{54}$.

In yeast, Ipl1 has been proposed to promote amphitelic kinetochoreattachment to microtubules by destabilizing syntelic attachments of SISTER CHROMATIDS ${ }^{55}$. This might be especially important in S. cerevisiae, in which chromosomes are attached to nuclear microtubules for most of the cell cycle and replicated sister kinetochores enter mitosis attached to the same spindle pole body. How the kinase recognizes syntelic attachments is not clear, but it has been proposed that tension between amphitelically oriented sister kinetochores stretches them apart enough to separate microtubulebinding sites from Aurora $B$ that is sequestered in the inner centromere, which thereby limits the accessibility of the kinaseto itssubstrate ${ }^{55}$.

Aurora B also seems to have an important role in regulating kinetochore-microtubule interactions in higher eukaryotes. Interference with its function by RNA ${ }^{51}$, microinjection of function-blocking antibodies $^{56}$ or treatment with small-moleculeinhibitors ${ }^{57,58}$ all cause defects in chromosome CONGRESSION. One study found a significant increase in the frequency of syntelic attachments after treatment with an inhibitor ${ }^{58}$. Expression of a dominant-negative catalytically inactive version of Aurora B in NRK (normal rat kidney) cells disrupts microtubuleattachment and causes a loss of dynein and centromere protein E (CENP-E) from kinetochores ${ }^{59}$. These motor proteins are thought to be involved in kinetochore binding to microtubules and also in signalling kinetochoreattachment to the spindle assembly checkpoint.

Kinetochoretargets of Aurora kinases are best known in budding yeast, in which the essential kinetochore protein $\mathrm{N} \mathrm{dc10}$ was shown to be a substrate of I pl1 (REF. 60; see FIG.6). Other kinetochore-associated targets of Ipl1 phosphorylation includel pl1 itself; itstargeting/activating factor Sli15, which is the yeast homologue of INCEN P; Dam1, a member of a protein complex the association of which with theouter kinetochore seems to beregulated by Ipl1; and Cse4, a kinetochore-specific histone-H 3 variant ${ }^{41,61-63}$. On the basis of thesestudies, a consensus phosphorylation site for I pl1, as mentioned above, has been defined as ((R/K)X(T/S)(I/L/V); REF. 41). The consensus sequence for metazoan Aurora $B$ is likely to be similar, but where phosphoacceptor sites have been mapped, they are preceded by two basic residues ${ }^{64}$.

The mammalian kinetochore-specific histone $\mathrm{H} 3$ variant CEN P-A is a substrate of Aurora $B$ in mammalian cell $1 S^{65}$. Phosphorylation of CENP-A by Aurora B peaks in prometaphase ${ }^{65}$. Surprisingly, phosphorylation-site mutants show a delay in the late stages of cytokinesis. Why a kinetochore protein should show defects in completion of cytokinesis, a cytoskeleton/membrane event, is unclear. Furthermore, I NCEN P and Aurora-B localization is perturbed in the CEN P-A phosphomimetic mutant S7E for reasons that remain unclear.

Aurora B in chromosomecondensation and cohesion? Aurora-B kinases are responsiblefor one of the classic modifications of chromatin in mitosis - phosphorylation of histone 3 on S10 (REF. 66). This modification, which is conserved from yeast to vertebrates, is carried 
CONDENSIN

A complex of two SMC

(structural maintenance of chromosomes) proteins and three auxiliary subunits that can bind to and supercoil DNA. Condensin directs the binding of other non-histone proteinsto mitotic chromosomes, and although not essential for mitotic chromatin condensation, it is essential for thestructural integrity of mitotic chromosomes.

CHIASM ATA

Connections formed between homologous chromosomes that arethought to bethe point of theinterchangethat is involved in crossing over.

\section{COHESIN}

A protein complex that tethers sister chromatids together from the timethey are created (during DNA replication) until cohesin cleavage at the onset of anaphase.

CLEAVAGE FURROW A region of the plasma membranein higher eukaryotic cells that ingresses to separate thetwo daughter cells at cytokinesis; contraction in this region is driven by actin and myosin filament interaction.

INTERM EDIATE FILAM EN A cytoskeletal filament, typically $10 \mathrm{~nm}$ in diameter, that occurs in higher eukaryotic cells. The protein composition of intermediatefilaments varies between cell types. Examples of intermediate filament proteins arekeratins, vimentin and desmin. out by I pl 1 in budding yeast ${ }^{67}$ and Aurora B in metazoans ${ }^{44,51,67-71}$. Aurora B is also responsiblefor histoneH3 phosphorylation on S28 from prophase to metaphase ${ }^{72,73}$. In living NRK cells undergoing prophase, Aurora-B-GFP was seen all over the chromatin, although it was enriched on centromere ${ }^{52}$. It had long been predicted that these histone modifications might help to drive mitotic chromatin condensation; however, when this was measured, the degree of histone-H 3 S10 phosphorylation was found not to correlate with thelevel of chromatin condensation ${ }^{51}$.

Defectsin chromosomestructureand compaction are observed in Aurora-B-depleted D rosophila cell $s^{51,74}$, but theunderlying mechanism isnot known. Onepossibility is that Aurora activity might be required for CONDENSIN function. However, the relationship between thekinase and condensin is unclear. The condensin complex ${ }^{75}$ does not localize properly to chromosomes in D rosophila cells that lack active Aurora B (REF. 74) or in S. pombe that lacksArk1 (REF. 76). In C. elegans, condensin activity is independent of Aurora B in prometaphase, but becomes dependent on the kinase in metaphase ${ }^{77,78}$; in Xenopus extracts, Aurora B is not required for condensin binding or chromosome condensation ${ }^{71}$. Furthermore, in cells treated with theAurora-B inhibitor hesperadin ${ }^{58}$ ( see BOX 2), in which Aurora B localizes properly but is enzymatically inactive, the condensin complex localizes normally. There is no evidence so far that any of the condensin subunits is a substrate of Aurora B.

In metaphase I of $C$. elegans meiosis, Aurora B has been shown to localize to the paired arms of the homologous chromosomes distal to the CHIASMATA ${ }^{77,79}$. It is the selective release of cohesion in this region that permits terminalization of chiasmata and releases homologous chromosomes for proper segregation to the spindle poles in anaphasel. Aurora B could regulate this process by phosphorylating Rec8, a meiosis-specific subunit of the COHESIN complex ${ }^{77,79}$. Interestingly, the centromeric localization of theAurora-B binding partners INCENP and survivin (see below) has been shown to depend on cohesin function in chicken DT40 B lymphocytes ${ }^{30}$.

Aurora B isessential for cytokinesis. Overexpression of a catalytically inactive form of rat Aurora B (which was previously known as Aim1) prevents thelast steps of cytokinesis in a number of cell lines ${ }^{50}$. A similar phenotype is produced by exogenous expression of a dominant mutant of the chromosomal passenger IN CENP (see below) that lackstheAurora-B-binding domain (IN-box) and delocalizes the kinase ${ }^{81,82}$.

Depletion of Aurora B by RNAi in D rosophila tissueculture cells and in C. elegans confirmed a role for the kinasein thelatestages of cytokinesis $51,69,83,84$. In somecases, inactivation of Aurora B wasassociated with delocalization of thekinesin-likeprotein Pavarotti/CHO1/M KLP1/ZEN 4 (CHO1, Chinesehamster ovary monoclonal antibody 1 ; M KLP1, mitotic kinesin-like protein 1; ZEN 4, zygotic enclosure defective), which is essential for establishment of thecentral spindle during anaphase ${ }^{69,83,84}$. However, in one study, Pavarotti was correctly local ized after depletion of AuroraB in cellsin which cytokinesisfailed ${ }^{51}$.

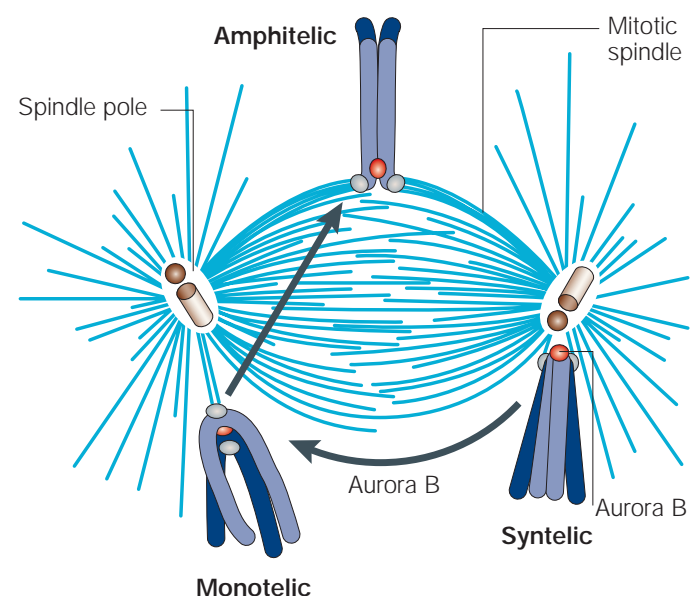

Figure 5 | Role of Aurora B in promoting chromosome biorientation on the mitotic spindle. Normally, the first attachment of chromosomes to the spindle microtubules (blue) is monotelic (one kinetochore bound, one kinetochore free). If both kinetochores attach to the same spindle pole (syntelic attachment), Aurora B in the inner centromere (red) promotes release of the bound microtubules, which therefore promotes the formation of monotelic attachments. Eventually, chromosomes become attached to both spindle poles (amphitelic attachment).

Other specific targets of Aurora B in the cLeavage FURROw include myosin II regulatory light chain ${ }^{85}$, vimentin ${ }^{86}$, desmin, and glial fibrillary acidic protein (GFAP; REF. 87; FIG.6). Although many of the phosphorylation sites in desmin and GFAP are also targeted by Rho kinase, one phosphorylation site in desmin is specifically phosphorylated by Aurora B. Phosphospecific antibodies showed that this phosphorylation occurs specifically at the cleavage furrow. Because phosphorylation has a dramatic effect on the stability of INTERM EDIATE FILAM ENTS, it was suggested that desmin phosphorylation by Aurora B could contribute to the destabilization of intermediatefilaments that is essential in the later stages of cytokinesi ${ }^{87}$.

Aurora B al so phosphorylates S387 of M gcRacGAP (which is known in C. elegans as Cyk4; REF. 88) in the midbody ${ }^{89}$. This phosphorylation significantly changes the specificity of M gcRacGAP from an activating protein for the small GT Pase Rac to an activator of RhoA. Phosphorylated M gcRacGAP colocalizes with Rho in the contractile ring. RhoA regulates actin polymerization and is essential for completion of cytokinesis ${ }^{90,91}$. In cells expressing a non-phosphorylatable form of M gcRacGAP, cleavage furrow ingression proceeds to a late stage but finally reverts without separation of the daughter cell $\mathrm{s}^{89}$, which indicates that any failure of Aurora B to phosphorylate M gcRacGAP would probably have the same effect.

Aurora B and thespindle-assembly checkpoint. Aurora $B$ and its associated proteins IN CEN P and survivin (see below) have recently emerged as important factors for the spindle-assembly checkpoint, a biochemical circuit that delays mitotic progression until all chromosomes 


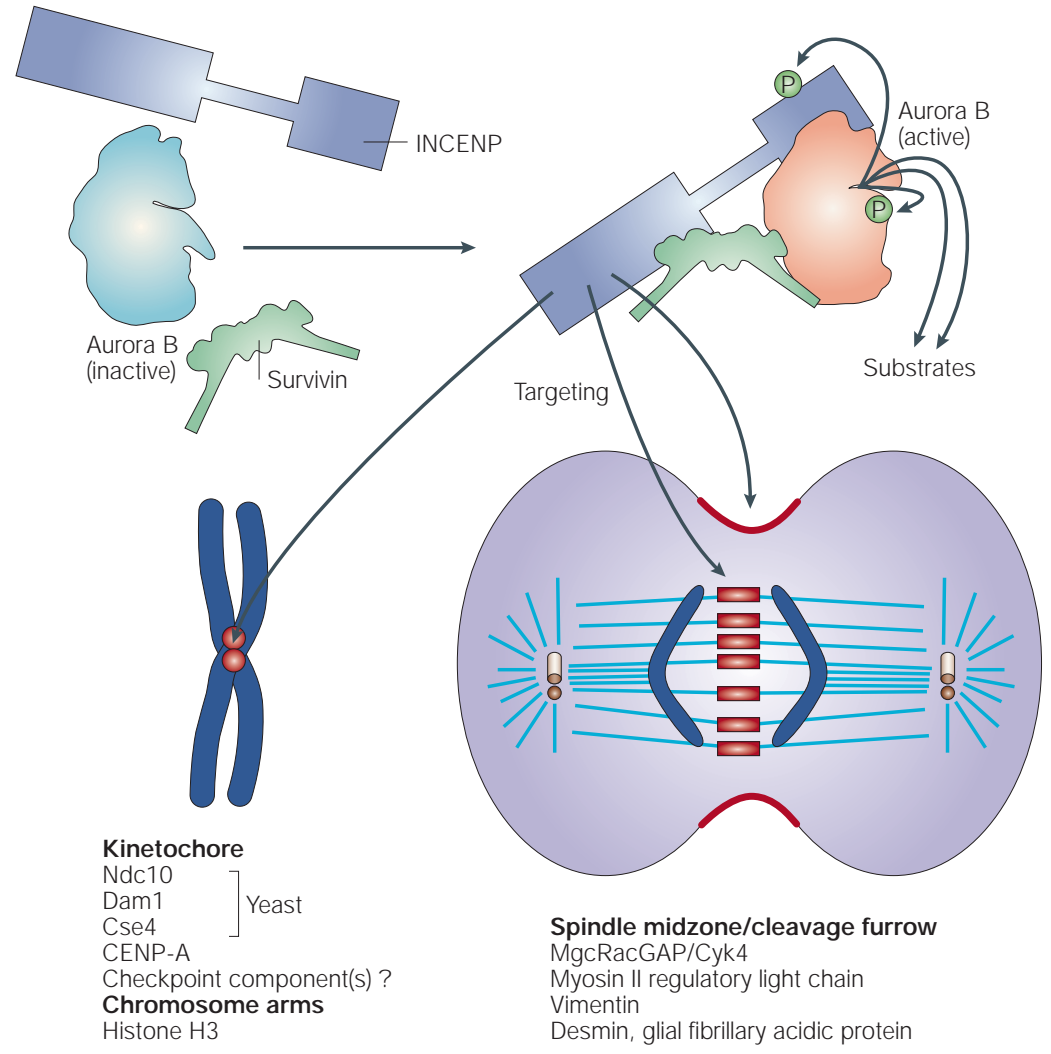

Figure 6 | Some of the known Aurora-B substrates in mitotic cells. Aurora B forms a complex - the chromosomal passenger complex - with inner centromere protein (INCENP) and survivin. Aurora-B phosphorylation of INCENP in turn promotes Aurora-B activity, as does survivin binding. INCENP targets the complex to centromeres (lower left part of figure) early in mitosis, and the spindle midzone and cleavage furrow late in mitosis (lower right part of figure). Some of the known substrates of Aurora B are shown - in particular, those that influence kinetochores, chromosome arms and the spindle midzone/cleavage furrow. CENP-A, centromere protein $\mathrm{A}$.
Aurora B'sfellow travellers, IN CEN P and survivin. In addition to being regulated by phosphorylation ${ }^{95}$, Aurora $B$ is also regulated by association with at least two other proteins, IN CEN P and survivin. Together, these three proteins are referred to as the chromosomalpassenger complex 6 . This complex is conserved from vertebrates to budding yeast and the proteinsinvolved are I pl 1, Sli15 and Bir ${ }^{41,61}$. In vertebrates it has now clearly been shown that each component of the complex is required for the proper localization of the two others ${ }^{82,83,93,96,97}$. So, I N CEN P and survivin apparently function to target thekinase, and themovement of the passenger complex from centromeres, to central spindle, to midbody, presumably reflects movement of the kinase to act on different substrates.

Both INCENP and survivin havea rolein activating the kinase. INCENP is a microtubule-binding protein ${ }^{98}$ that has a non-conserved amino-terminal region that is important for targeting the protein to chromosomes and centromeres ${ }^{99}$, and a highly conserved carboxyterminal motif, the IN-box, that is responsible for binding Aurora B (REFS64,100). I NCEN P is both a substrate and a positive regulator of the kinase. It is phosphorylated by Aurora B in the IN-box $41,64,101$ and this phosphorylation enhances the activity of the kinase. Although the mechanism is not yet known, recent results indicate that IN CEN P phosphorylation might alter the protein structure in the region of the IN -box, and this might somehow contribute to thestimulation of kinase activity ${ }^{64}$.

Survivin is a small protein containing a BIR (baculovirus inhibitor of apoptosis (IAP) repeat) domain, a $\mathrm{Zn}^{2+}$-finger motif that is found in theIAP proteins, which are important regulators of the apoptotic pathway ${ }^{102}$. Evidence concerning whether survivin has an important rolein apoptosis regulation is controversial, but genetic experiments in mice ${ }^{103}$ and RNAi studies indicate that the primary role of the protein is in mitosis, in which it is required for the localization of both Aurora B and INCEN P93. Xenopus survivin binds to Aurora $B$ and enhances its activity in vitro ${ }^{97}$, although human survivin does not stimulate the kinaseactivity in similar assays ${ }^{64}$. Treatment of cells with survivin antisense RNA results in decreased Aurora-B activity and mislocalization of the kinase ${ }^{104}$. The $S$. pombesurvivin homologue Cut17/Bir1 is required for Ark1 localization $^{105}$ and activity ${ }^{76}$.

Three other chromosomal passengers have recently been characterized. One of them - TD-60 - which colocalizes exactly with IN CEN P in cell $s^{106}$, is apparently a guanine nucleotide exchange factor ${ }^{107}$. This raises the possibility that a small G-protein - probably Racl - might be involved in the regulation of the activity of the passenger complex that contains Aurora-B kinase. The second passenger, inner centromere Kinl stimulator (ICIS), is an inner centromere protein that seems to regulate the microtubule-disassembly activity of mitotic centromere-associated kinesin (MCAK) ${ }^{108}$. It is possible that ICIS is a substrate of Aurora B. Genetic analysis in C. elegans has recently identified an additional component of the chromosomal passenger 
Box 2 | New inhibitors of Aurora-B kinase

Two recent studies using small-molecule inhibitors of Aurora kinases have opened new possibilities for thestudy of the function of these kinases.

ZM 447439 inhibits Aurora A and B with IC $C_{50}$ s of 0.11 and $0.13 \mu \mathrm{M}$, respectively ${ }^{57}$. The TC $_{50}$ for most other kinases tested was $>10 \mu \mathrm{M}$, but lymphocyte-specific protein tyrosine kinase (LCK) was inhibited at $0.88 \mu \mathrm{M}$. Phosphorylation of histone 3 is abolished in human cells treated with ZM 447439. Furthermore, although a normal bipolar spindle forms and kinetochores attach to microtubules, chromosomes do not align properly at a metaphase plate. Although drug treatment does not affect the localization of Aurora A, Aurora B or survivin, it substantially reduces the levels of BubRI, Mad2 and centromere protein E (CEN P-E) at kinetochores and compromises thespindle-assembly checkpoint. Effects on cytokinesis were not examined in detail. These phenotypes resemble those seen after RNA interference (RNAi)mediated suppression of Aurora B, but not Aurora A, although the Aurora-B RNAi phenotype is more severe. Why the cells do not show the phenotypes that are expected for inhibition of Aurora A (for example, failure of centrosome maturation) is not known.

Hesperadin, a structurally distinct inhibitor, produces similar effects in a variety of cell types ${ }^{58}$. In addition to Aurora B, hesperadin at $1 \mu \mathrm{M}$ also significantly inhibits at least six other kinases, including checkpoint kinase 1 (CHK1), LCK, mitogen-activated protein kinase (MAPK)-associated protein kinase 1 (MAPKAP-K1), MAPK kinase 1 (MKK1), phosphorylase kinase (PHK) and AM P-activated protein kinase (AM PK). The authors argue that the drug is specific for Aurora B because, at 0.2-1 $\mu \mathrm{M}$, its effects on cultured cells resemblethose seen after Aurora-B RNAi treatment. Like ZM 447439, hesperadin inhibits the spindle-assembly checkpoint response to Taxol, but not nocodazole (at least over a 3-hour period), as expected, given the involvement of Aurora B in thetension-sensitive arm of the spindle-assemblycheckpoint pathway. A detailed analysis of microtubule attachments in cycling and hesperadin-treated cells showed that the drug causes a significant increase in the number of syntelic chromosome attachments seen, apparently at the cost of monotelic attachments. Overall, the data support a model in which the target of hesperadin (presumably Aurora B) helps promote normal chromosomealignment in mitosis by destabilizing syntelic attachments, as had been previously proposed for I pl1 in budding yeast ${ }^{55}$.

complex - chromosome segregation and cytokinesis defective (CSC-1; REF. 109). CSC-1 binds to C. elegans BIR-1/survivin and can form a four-way complex with INCENP and Aurora B. The role of CSC-1 in this complex is not known. To date, CSC-1 has not been detected in species other than nematodes.

\section{Aurora kinases and cancer}

Aurora kinases are of great interest for cancer researchers. The genes encoding the three human Aurora kinases map to regions that are affected by chromosomal abnormalities in different cancer types, and overexpression of each of thethree human Auroras has been detected in tumour cell lines, $8,15,110,111$.

Aurora A. To date, most interest has focused on AuroraA, which fits the criteria to beclassified as a bona fide oncogene (FIG. 7). Human Aurora A maps to chromosomal region 20q13.2, which is amplified in a number of cancer cell lines and primary tumours $8,112,113$. Furthermore, mouse NIH -3T3 cellstransfected with AuroraA giverise to tumours when injected into nude mice ${ }^{8,39,112}$. This requires the catalytic activity of the kinase ${ }^{8}$.

How might the inappropriateexpression of AuroraA transform cells? One possibility is that the kinase somehow uncouples centrosome duplication from the cell cycle, thereby causing centrosomeamplification. It is now almost 100 years since the proposal by Boveri that cancer might be caused by centrosomal abnormalities $^{114}$. The relationship between such abnormalities and cancer has now been clearly shown ${ }^{115}$, and considerable efforts have been focused on determining whether the centrosomal phenotypes area cause or consequence of the canceroustransformation.
Two recent studies indicate that overexpression of Aurora A is unlikely to trigger centrosome amplification directly, but instead leads to mitotic abnormalities that culminate in the failure of cytokinesis and the production of tetraploid cells ${ }^{116,117}$. Aurora A is not unique in this regard, as overexpression of Aurora $B$ or Polo-like kinase (PIk1) al so causes cytokinesis failure and centrosome duplication ${ }^{110,116}$. Remarkably, this phenotype did not require that the two Aurora kinases be catalytically active - catalytically inactive mutants were as capable as, and in some cases more capable than, the kinase-active forms of inducing cytokinesis failure and centrosome amplification ${ }^{116}$. The mechanism of this cytokinesis failure is not known, but overexpression of Aurora $A$ has been shown to perturb the spindle-assembly checkpoint ${ }^{117}$. This raises the possibility that abnormal spindle arrangements caused by excessive kinase activity that would normally trigger checkpoint activation are, in this case, unable to cause a mitotic arrest, with the consequences that cells exit mitosis with chromosomal and spindle arrangements that preclude successful cytokinesis.

Aurora-induced mitotic abnormalities are exacerbated in cells that lack p53 (REF. 116). This is partly because 553 can bind to Aurora A and directly inactivate its kinase function ${ }^{118}$. In addition, cells that fail in cytokinesis normally arrest in the G1 phase of thenext cell cycle as the result of a p53-dependent checkpoint that seems to detect tetraploidy and block cell-cycle progression ${ }^{119-123}$. It could be, therefore, that in the absence of p53, cells in which overexpression of Aurora kinases has led to cytokinesis failurecan then proceed through the cell cycle, undergo subsequent error-prone 


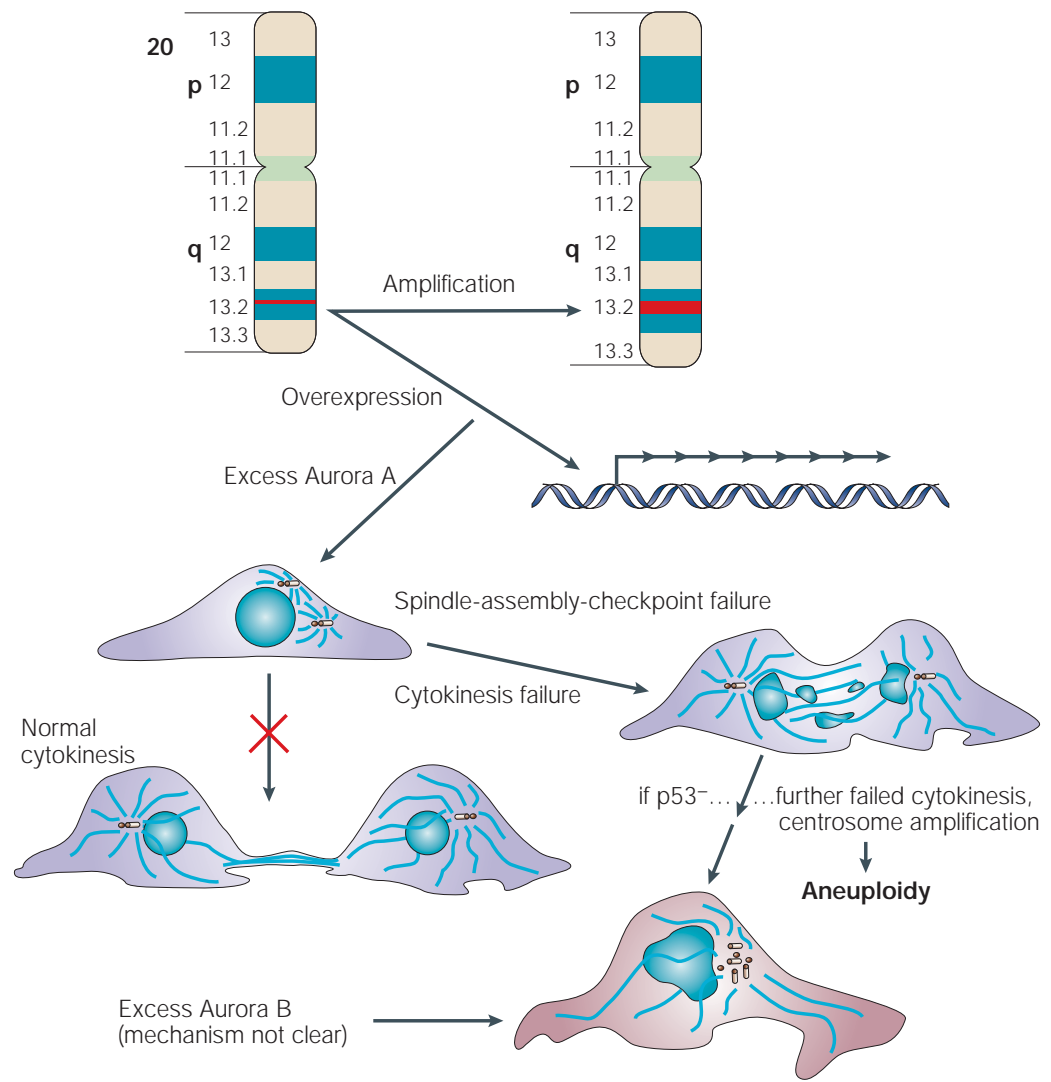

Figure 7 | Aurora A and cancer. Overexpression of Aurora A, either as a consequence of gene amplification or by other means, disrupts mitotic progression, apparently by blocking the ability of chromosomes to achieve a normal orientation on the spindle. Despite the difficulties with chromosome alignment, these cells exit mitosis because Aurora-A overexpression also inactivates the spindle-assembly checkpoint. The cells ultimately fail in cytokinesis, producing tetraploid progeny. If the cells also lack p53 (p53-), they continue through subsequent cell cycles, ultimately becoming polyploid and, eventually, aneuploid, with amplified centrosomes. Aurora A could also potentially contribute to carcinogenesis in other ways that are not shown here. Dysregulation of Aurora-B kinase might also lead to ANEUPLOIDY and cancer (lower left), but the mechanism is much less explored than that for Aurora A.

ANEUPLOIDY

The ploidy of a cell refers to the number of sets of chromosomes that it contains. Aneuploid karyotypes are those whose chromosomecomplements are not asimplemultiple of the haploid set. mitosis, and generate aneuploid progeny. Whether cytokinesis failures represent the only route to centrosomeamplification is a very important topic for future experimentation.

Why should the overexpression of Aurora A perturb mitosis and produce an effect that actually resembles that seen with a dominant-negative catalytically inactive Aurora-B mutant ${ }^{50}$ ? As described above, thetwo kinases sharea high degree of homology in the kinase domain. In fact, both can phosphorylatehistone H 3 at S10 (REF.70), and Aurora A has been shown to bind survivin in a ternary complex with the GTPase-activating protein RasGAP (REF. 124). One attractive possibility is that overexpressed Aurora A might competewith Aurora B for substrates and/or regulators.

Aurora B. Aurora B has also been implicated in cancer. $\mathrm{CHO}$ cells overexpressing Aurora B show elevated levels of phosphorylated histone $\mathrm{H} 3$ and defects in chromosome segregation and cytokinesis $s^{125}$. The progeny of these cells are aneuploid and can produce aggressive tumours in mice. Increased levels of phosphorylation of histone $\mathrm{H} 3$ werealso shown to correlatewith overexpression of Aurora B in somehuman colorectal tumour cell lines ${ }^{125}$.

The Aurora theme: location, location, location Aurora kinases have exploded on the scene in the past five years, and are now recognized as leading players in the orchestration of mitotic events. Aurora-A kinases are particularly important for regulation of microtubule nucleation at spindle poles, and are subject to a complex network of controls that include phosphorylation, association with TPX 2 and destruction by the APC/C. Aurora B kinases are essential for chromosome condensation, kinetochorefunction, cytokinesis and the proper function of the spindle-assembly checkpoint when spindle tension is perturbed. Their regulation involves association with the chromosomal passenger proteins INCEN P and survivin, which are important both for targeting and activation of the kinase. Littleis known about Aurora-C kinases, which are expressed at high levels only in the testis, and comprise a particularly interesting subject for future studies. The single Aurora kinases of the budding and fission yeasts are particularly important for kinetochore-microtubule attachments and spindle-assembly checkpoint function, which are activities that are most closely associated with Aurora-B kinases. However, in a study in which kinase domains were swapped, only the kinase domain of Aurora A and not that of Aurora B could substitute for I pl1 in budding yeast ${ }^{8}$, indicating that I $\mathrm{pl} 1$ is probably required for both Aurora-A and -B functions. Adding to the interest in these kinases is the fact that Aurora kinase overexpression has been observed in cancer, and Aurora A can function as a bona fide oncogene.

The best known cell-cycle regulatory kinases are cyclin-dependent kinases (CDKs), so named because they require the association of a cyclin subunit for catalytic activity ${ }^{126,127}$. Binding of the cyclin subunit to the CDKs changes the kinase structure, preparing it for activation by CDK-activating kinase (CAK) and promoting the binding of substrates ${ }^{128}$.

It now seems that the Aurora kinases are similar, but different, to CDKs. Bacterially expressed Aurora A and B are catalytically active, so they do not absolutely require the binding of associated subunits for activity. However, they aremuch moreactive when associated with auxiliary factors. In the case of Aurora B (and the yeast Auroras), thesefactors are INCENP and survivin ${ }^{61,97,101}$ In the case of Aurora A, TPX2 is one such factor ${ }^{28,32}$. Significantly, thehomology with CDKs breaks down when the role of these auxiliary subunits is considered. AuroraA does not absolutely requirean external activating kinase, although PKA and M SK 1 (mitogen- and stress-activated protein kinase) can do thi ${ }^{32}$. In fact, TPX2 seems to enableAurora $A$ to activateitself and to resist inactivation by PP1 (REF. 32). It will beimportant to demonstratein the future whether a similar mechanism operates for Aurora B and INCEN P/survivin. 
Interestingly, both TPX 2 and IN CENP are substrates of their respective kinases ${ }^{61,97,101,129}$ and I N CEN P phosphorylation has been shown to be required for thestimulation of kinase activity ${ }^{101}$.

If therole of INCEN P/survivin and TPX 2 in kinase activation is considered further, a picture emerges of the Auroras as 'journeyman kinases' that travel from location to location in the cell to do their work. In addition to promoting kinase activation, I NCENP/survivin and TPX 2 also direct the location of their respective Aurora kinases in the cell $25,28,82,83,96$. This provides a very powerful means of regulation, and might beessential to the ability of these kinases to choreograph mitotic events. If the auxiliary factors were essential for both function and targeting, this would enable the kinase activity to be moved from place to place within the mitotic cell with great temporal and spatial precision. For example, Aurora B moves from the entire chromosome, to the centromere, to the central spindle and equatorial cortex as cells traverse mitosis $s^{50,82}$. The auxiliary factors also provide rich targets for regulatory activities, and we predict that deciphering the regulation of their movements will prove to beessential for the complete understanding of Aurora kinase activity. Clearly, much new territory remains to be explored in the world of Aurora kinases.
1. Glover, D. M., Leibowitz, M. H., McLean, D. A. \& Parry, H. Mutations in aurora prevent centrosome separation leading to the formation of monopolar spindles. Cell 81, 95-105 (1995).

2. Sunkel, C. E. \& Glover, D. M. Polo, a mitotic mutant of Drosophila displaying abnormal spindle poles. J . Cell Sci. 89, 25-38 (1988)

3. Chan, C. S. \& Botstein, D. Isolation and characterization of chromosome-gain and increase-in-ploidy mutants in yeast. Genetics 135, 677-691 (1993)

4. Petersen, J., Paris, J., Willer, M., Philippe, M. \& Hagan, I. M. The S. pombe aurora-related kinase Ark 1 associates with mitotic structures in a stage dependent manner and is required for chromosome segregation. J. Cell Sci. 114 4371-4384 (2001).

5. Leverson, J. D., Huang, H. K., Forsburg, S. L. \& Hunter, T. The Schizosaccharomyces pombe Aurora-related kinase Ark1 interacts with the innercentromere protein Picl and mediates chromosome segregation and cytokinesis. Mol. Biol. Cell 13, 1132-1143 (2002).

6. Adams, R. R., Carmena, M. \& Earnshaw, W. C. Chromosomal passengers and the (Aurora) ABCs of mitosis. Trends Cell Biol. 11, 49-54 (2001).

7. Nigg, E. A. Mitotic kinases as regulators of cell division and its checkpoints. Nature Rev. Mol. Cell. Biol. 2, 21-32 (2001).

8. Bischoff, J. R. et al. A homologue of Drosophila aurora kinase is oncogenic and amplified in human colorectal cancers. EMBO J . 17, 3052-3065 (1998). Provides one of the first insights into the link between Aurora kinases and carcinogenesis.

9. Giet, R. \& Prigent, C. Aurora/lplip-related kinases, a new oncogenic family of mitotic serine-threonine kinases. J. Cell Sci. 112, 3591-3601 (1999).

10. Cheetham, G. M. et al. Crystal structure of aurora-2, an oncogenic serine/threonine kinase. J. Biol. Chem. 277, 42419-42422 (2002)

11. Nowakowski, J. et al. Structures of the cancer-related Aurora-A, FAK, and EphA2 protein kinases from nanovolume crystallography. Structure 10, 1659-1667 (2002).

12. Earnshaw, W. C. \& Bernat, R. L. Chromosomal passengers: towards an integrated view of mitosis. Chromosoma $\mathbf{1 0 0}$ 139-146 (1990).

13. Bemard, M., Sanseau, P., Henry, C., Couturier, A. \& Prigent, C. Cloning of STK13, a third human protein kinase related to Drosophila aurora and budding yeast Ipl1 that maps on chromosome 19q13.3-ter. Genomics 53, 406-409 (1998).

14. Tseng, T. C., Chen, S. H., Hsu, Y. P. \& Tang, T. K. Protein kinase profile of sperm and eggs: cloning and characterization of two novel testis-specific protein kinases (AIE 1, AIE2) related to yeast and fly chromosome segregation regulators. DNA Cell Biol. 17, 823-833 (1998).

15. Kimura, M., Matsuda, Y., Yoshioka, T. \& Okano, Y. Cell cycle-dependent expression and centrosome localization of a third human aurora/Ipl1-related protein kinase, AIK3. J. Biol. Chem. 274, 7334-7340 (1999)

16. Hu, H. M., Chuang, C. K., Lee, M. J ., Tseng, T. C. \& Tang, T. K. Genomic organization, expression, and chromosome localization of a third aurora-related kinase gene, Aie1. DNA Cell Biol. 19, 679-688 (2000).

17. Schumacher, J. M., Ashcroft, N., Donovan, P. J . \& Golden, A A highly conserved centrosomal kinase, AIR-1, is required for accurate cell cycle progression and segregation of developmental factors in Caenorhabditis elegans embryos. Development 125, 4391-4402 (1998).

18. Roghi, C. et al. The Xenopus protein kinase $\mathrm{pEg} 2$ associates with the centrosome in a cell cycle-dependent manner binds to the spindle microtubules and is involved in bipolar mitotic spindle assembly. I. Cell Sci. 111, 557-572 (1998).

19. Sugimoto, $\mathrm{K}$. et al. Molecular dynamics of aurora-A kinase in living mitotic cells simultaneously visualized with histone $\mathrm{H} 3$ and nuclear membrane protein importin $\alpha$. Cell Struct. Funct. 27, 457-467 (2002).

20. Berdnik, D. \& Knoblich, J. A. Drosophila Aurora-A is required for centrosome maturation and actin-dependent asymmetric protein localization during mitosis. Curr. Biol. 12 , 640-647 (2002)

This describes a role for Aurora $A$ in asymmetric cell division and examines the dynamics of Aurora-A association with centrosomes.

21. Wittmann, T., Wilm, M., Karsenti, E. \& Vernos, I. TPX2, a novel Xenopus MAP involved in spindle pole organization. J. Cell Biol. 149, 1405-1418 (2000).

22. Gruss, O. J . et al. Chromosome-induced microtubule assembly mediated by TPX2 is required for spindle formation in HeLa cells. Nature Cell Biol. 4, 871-879 (2002)

23. Giet, R. \& Prigent, C. The non-catalytic domain of the Xenopus laevis aurora A kinase localises the protein to the centrosome. J . Cell Sci. 114, 2095-2104 (2001).

24. Giet, R. et al. Drosophila Aurora A kinase is required to localize D-TACC to centrosomes and to regulate astral microtubules. J. Cell Biol. 156, 437-451 (2002). RNAi analysis confirms a role for Aurora A in centrosome maturation and separation.

25. Kufer, T. A. et al. Human TPX2 is required for targeting Aurora-A kinase to the spindle. J. Cell Biol. 158, 617-623 (2002).

26. Hannak, E., Kirkham, M., Hyman, A. A. \& Oegema, K. Aurora-A kinase is required for centrosome maturation in Caenorhabditis elegans. J. Cell Biol. 155, 1109-1116 (2001).

27. Giet, R., Uzbekov, R., Cubizolles, F., Le Guellec, K. \& Prigent, $C$. The Xenopus laevis aurora-related protein kinas pEg2 associates with and phosphorylates the kinesinrelated protein XIEg5. J . Biol. Chem. 274, 15005-15013 (1999).

28. Tsai, M. Y. et al. A Ran signalling pathway mediated by the mitotic kinase Aurora A in spindle assembly. Nature Cell Biol. 5, 242-248 (2003)

This study shows that TPX2 activates Aurora-A kinase, and proposes that Aurora A might be an important downstream target of Ran-GTP in promoting mitotic spindle assembly.

29. Wilde, A. \& Zheng, Y. Stimulation of microtubule aster formation and spindle assembly by the small GTPase Ran. Science 284, 1359-1362 (1999).

30. Kalab, P., Pu, R. T. \& Dasso, M. The ran GTPase regulates mitotic spindle assembly. Curr. Biol. 9, 481-484 (1999).

31. Carazo-Salas, R. E. et al. Generation of GTP-bound Ran by $\mathrm{RCC} 1$ is required for chromatin-induced mitotic spindle formation. Nature 400, 178-181 (1999).

32. Eyers, P. A., Erikson, E., Chen, L. G. \& Maller, J . L. A novel mechanism for activation of the protein kinase aurora $a$. Curr. Biol. 13, 691-697 (2003).

This study shows that TPX2 activates Aurora-A kinase in part by blocking the inactivation of the kinase by protein phosphatase 1.

33. Andresson, T. \& Ruderman, J . V. The kinase Eg2 is a component of the Xenopus oocyte progesterone-activated signaling pathway. EMBO J . 17, 5627-5637 (1998).

34. Mendez, R. et al. Phosphorylation of CPE binding factor by Eg2 regulates translation of c-mos mRNA. Nature 404, 302-307 (2000).
This describes the involvement of Aurora A in cellcycle regulation by regulating poly(A)-dependent translation.

35. Frank-Vaillant, M. et al. Progesterone regulates the accumulation and the activation of Eg2 kinase in Xenopus oocytes. J . Cell Sci. 113, 1127-1138 (2000)

36. Maton, $\mathrm{G}$. et al. $\mathrm{Cdc} 2-\mathrm{Cyclin} \mathrm{B}$ triggers $\mathrm{H} 3$ kinase activation of Aurora-A in Xenopus oocytes. J . Biol. Chem. 278, 21439-21449 (2003).

37. Schumacher, J. M., Golden, A. \& Donovan, P. J . AlR-2 An Aurora//pl1-related protein kinase associated with chromosomes and midbody microtubules is required for polar body extrusion and cytokinesis in Caenorhabditis elegans embryos. J. Cell Biol. 143, 1635-1646 (1998).

38. Castro, A., Mandart, E., Lorca, T. \& Galas, S. Involvement of Aurora A kinase during meiosis I-II transition in Xenopus oocytes. J . Biol. Chem. 278, 2236-2241 (2003).

39. Littlepage, L. E. et al. Identification of phosphorylated residues that affect the activity of the mitotic kinase Aurora-A. Proc. Natl Acad. Sci. USA 99, 15440-15445 (2002).

Systematic analysis of the residues that are relevan for regulation of Aurora-A kinase.

40. Walter, A. O., Seghezzi, W., Korver, W., Sheung, I \& \& Lees, E. The mitotic serine/threonine kinase Aurora2/AIK is regulated by phosphorylation and degradation. Oncogene $\mathbf{1 9}$ 4906-4916 (2000).

41. Cheeseman, I. M. et al. Phospho-regulation of kinetochoremicrotubule attachments by the Aurora kinase Ipl1p. Cell 111, 163-172 (2002)

This is the most thorough study yet of the protein targets of the Aurora kinase Ipl1.

42. Francisco, L., Wang, W. \& Chan, C. S. Type 1 protein phosphatase acts in opposition to IpL1 protein kinase in regulating yeast chromosome segregation. Mol. Cell. Biol. 14. 4731-4740 (1994).

43. Katayama, H., Zhou, H., Li, Q., Tatsuka, M. \& Sen, S Interaction and feedback regulation between STK15/BTAK/Aurora-A kinase and protein phosphatase 1 through mitotic cell division cycle. J . Biol. Chem. 276, 46219-46224 (2001).

44. Murnion, M. E. et al. Chromatin-associated protein phosphatase 1 regulates Aurora- $\mathrm{B}$ and histone $\mathrm{H} 3$ phosphorylation. J. Biol. Chem. 276, 26656-26665 (2001).

45. Honda, K. et al. Degradation of human Aurora2 protein kinase by the anaphase-promoting complex-ubiquitinproteasome pathway. Oncogene 19, 2812-2819 (2000).

46. Castro, A. et al. APC/Fizzy-Related targets Aurora-A kinase for proteolysis. EMBO Rep. 3, 457-462 (2002).

47. Castro, A. et al. The D-Box-activating domain (DAD) is a new proteolysis signal that stimulates the silent $D-B$ ox sequence of Aurora-A. EMBO Rep. 3, 1209-1214 (2002).

48. Littlepage, L. E. \& Ruderman, J . V. Identification of a new $A P C / C$ recognition domain, the $A$ box, which is required for the Cdh1-dependent destruction of the kinase Aurora-A during mitotic exit. Genes Dev. 16, 2274-2285 (2002). References $\mathbf{4 6}$ and $\mathbf{4 8}$ do a good job of dissecting the complexities of Aurora-A regulation by the APC/C.

49. Kiat, L. S., Hui, K. M. \& Gopalan, G. Aurora-A kinase interacting protein (AIP), a novel negative regulator of human Aurora-A kinase. J. Biol. Chem. 277, 45558-45565 (2002).

50. Terada, Y. et al. AlM-1: a mammalian midbody-associated protein required for cytokinesis. EMBO J . 17, 667-676 (1998).

One of the first reports showing the requirement of Aurora B for cytokinesis. 
51. Adams, R. R., Maiato, H., Earnshaw, W. C. \& Carmena, M. Essential roles of Drosophila inner centromere protein (INCENP) and Aurora-B in histone $\mathrm{H} 3$ phosphorylation, metaphase chromosome alignment, kinetochore disjunction, and chromosome segregation. J . Cell Biol. 153 865-880 (2001)

52. Murata-Hori, M., Tatsuka, M. \& Wang, Y. L. Probing the dynamics and functions of Aurora $B$ kinase in living cells during mitosis and cytokinesis. Mol. Biol. Cell 13, 1099-1108 (2002)

Examines the dynamic behaviour of Aurora-B kinase in vivo using green-fluorescent-protein-labelled protein.

53. Nicklas, R. B. \& Staehly, C. A. Chromosom micromanipulation. I. The mechanics of chromosome attachment to the spindle. Chromosoma 21, 1-16 (1967).

54. Nicklas, R. B., Wards, S. C. \& Gorbsky, G. J . Kinetochore chemistry is sensitive to tension and may link mitotic forces for a cell cycle checkpoint I. Cell Biol. 130, 929-939 (1995).

55. Tanaka, T. U. et al. Evidence that the Ipl1-Sli15 (Aurora kinase-INCENP) complex promotes chromosome bi-orientation by altering kinetochore-spindle pole connections. Cell 108, 317-329 (2002). This paper was the first to propose that Ipl1 might be involved in the resolution of syntelic chromsome attachments.

56. Kallio, M. J., McCleland, M. L., Stukenberg, P. T. \& Gorbsky, G. J . Inhibition of aurora B kinase blocks chromosome segregation, overrides the spindle checkpoint, and perturbs microtubule dynamics in mitosis Curr. Biol. 12, 900-905 (2002).

Antibody injection is used to identify some of the many functions of Aurora B in mammalian cells.

57. Ditchfield, C. et al. Aurora B couples chromosome alignment with anaphase by targeting BubR1, Mad2 and
Cenp-E to kinetochores. I. Cell Biol. 161, 267-280 (2003). This description of a small-molecule inhibitor of Aurora B identifies a role for the kinase in the spindleassembly checkpoint when the checkpoint is activated in the presence of microtubules by altering tension within the spindle.

58. Hauf, $\mathrm{S}$. et al. The small molecule Hesperadin reveals a role for Aurora B in correcting kinetochore-microtubule attachment and in maintaining the spindle assembly checkpoint. J . Cell Biol. 161, 281-294 (2003). This description of a second small-molecule inhibitor of Aurora B provides the best evidence to date that the kinase is involved in the resolution of syntelic chromosomal attachments during prometaphase.

59. Murata-Hori, M. \& Wang, Y. The kinase activity of Aurora B is required for kinetochore-microtubule interactions during mitosis. Curr. Biol. 12, 894-899 (2002)

60. Biggins, S. et al. The conserved protein kinase Ipl1 regulates microtubule binding to kinetochores in budding yeast. Genes Dev. 13, 532-544 (1999).

61. Kang, J . et al. Functional cooperation of Dam1, Ipl1, and the inner centromere protein (INCENP)-related protein Sli15 during chromosome segregation. J. Cell Biol. 155, 763-774 (2001).

62. Buvelot, S., Tatsutani, S. Y., Vermaak, D. \& Biggins, S. The budding yeast Ipl1/Aurora protein kinase regulates mitotic spindle disassembly. J. Cell Biol. 160, 329-339 (2003).

63. Shang, C. et al. Kinetochore protein interactions and their regulation by the Aurora kinase Ipllp. Mol. Biol. Cell 14 3342-3355 (2003)

64. Honda, R., Korner, R. \& Nigg, E. A. Exploring the functional interactions between Aurora B, INCENP, and survivin in mitosis. Mol. Biol. Cell 14, 3325-3341 (2003). A thorough study of the regulation of Aurora-B kinase activity by INCENP and survivin.

65. Zeitlin, S. G., Shelby, R. D. \& Sullivan, K. F. CENP-A is phosphorylated by Aurora B kinase and plays an unexpected role in completion of cytokinesis. J. Cell. Biol. 155, 1147-1157 (2001).

66. Gurley, L. R., D'Anna, J. A., Barham, S. S., Deaven, L. L. \& Tobey, R. A. Histone phosphorylation and chromatin structure during mitosis in Chinese hamster cells. Eur. J. Biochem. 84, 1-15 (1978).

67. Hsu, J. Y. et al. Mitotic phosphorylation of histone $\mathrm{H}_{3}$ is governed by Ipl1/aurora kinase and Glc7/PP1 phosphatase in budding yeast and nematodes. Cell 102, 279-291 (2000).

68. Speliotes, E. K., Uren, A., Vaux, D. \& Horvitz, H. R. The survivin-like $C$. elegans BIR-1 protein acts with the Auroralike kinase AIR-2 to affect chromosomes and the spindle midzone Mol. Cell 6, 211-223 (2000).

69. Giet, R. \& Glover, D. M. Drosophila Aurora B kinase is required for histone $\mathrm{H} 3$ phosphorylation and condens recruitment during chromosome condensation and to organize the central spindle during cytokinesis. J . Cell Biol. 152, 669-681 (2001)
70. Crosio, C. et al. Mitotic phosphorylation of histone H3: spatio-temporal regulation by mammalian Aurora kinases. Mol. Cell. Biol. 22, 874-885 (2002)

71. MacCallum, D. E., Losada, A., Kobayashi, R. \& Hirano, T. ISWI remodeling complexes in Xenopus egg extracts: identification as major chromosomal components that are regulated by INCENP-aurora B. Mol. Biol. Cell 13, 25-39 (2002).

72. Goto, H. et al. Identification of a novel phosphorylation site on histone $\mathrm{H} 3$ coupled with mitotic chromosome condensation. J. Biol. Chem. 274, 25543-25549 (1999).

73. Goto, H., Yasui, Y., Nigg, E. A. \& Inagaki, M. Aurora-B phosphorylates $H$ istone $\mathrm{H} 3$ at serine28 with regard to the mitotic chromosome condensation. Genes Cells 7, 11-17 (2002).

74. Giet, R. \& Prigent, C. The Xenopus laevis aurora/lp11prelated kinase $p E g 2$ participates in the stability of the bipola mitotic spindle. Exp. Cell Res. 258, 145-151 (2000).

75. Hirano, T. The ABC S of SMC proteins: two-armed ATPases for chromosome condensation, cohesion, and repair. Genes Dev. 16, 399-414 (2002).

76. Petersen, J. \& Hagan, I. M. S. pombe Aurora kinase/survivin is required for chromosome condensation and the spindle checkpoint attachment response. Curr. Biol. 13, 590-597 (2003).

\section{This study uses yeast genetics to show a role for} Aurora B and its associated protein survivin in the spindle-assembly checkpoint.

77. Kaitna, S., Pasierbek, P., J antsch, M., Loidl, J \& G Glotzer, M. The Aurora B kinase AIR-2 regulates kinetochores during mitosis and is required for separation of homologous chromosomes during meiosis. Curr. Biol. 12, 798-812 (2002).

78. Hagstrom, K. A., Holmes, V. F., Cozzarelli, N. R. \& Meyer, B. J $C$. elegans condensin promotes mitotic chromosome architecture, centromere organization, and sister chromatic segregation during mitosis and meiosis. Genes Dev. 16, 729-742 (2002)

79. Rogers, E., Bishop, J. D., Waddle, J. A., Schumacher, J. M. $\&$ Lin, R. The aurora kinase AIR-2 functions in the release of chromosome cohesion in Caenorhabditis elegans meiosis. J. Cell Biol. 157, 219-229 (2002).

\section{This provides important insights into the role of} Aurora B in chromosome segregation in meiosis.

80. Sonoda, E. et al. Scc1/Rad21/Mcd1 is required for sister chromatid cohesion and kinetochore function in vertebrate cells. Dev. Cell 1, 759-770 (2001)

81. Mackay, A. M., Ainsztein, A., Eckley, D. M. \& Earnshaw, W. C. A dominant mutant of inner centromere protein (INCENP), a chromosomal protein, disrupts prometaphase congression and cytokinesis. J. Cell Biol. 140, 991-1002 (1998)

82. Adams, R. R. et al. INCENP binds the aurora-related kinase AIRK2 and is required to target it to chromosomes, the central spindle and cleavage furrow. Curr. Biol 10, 1075-1078 (2000)

83. Kaitna, S., Mendoza, M., J antsch-Plunger, V. \& Glotzer, M. Incenp and an Aurora-like kinase form a complex essential for chromosome segregation and efficient completion of cytokinesis. Curr. Biol. 10, 1172-1181 (2000).

84. Severson, A. F., Hamill, D. R., Carter, J . C., Schumacher, J $\&$ Bowerman, B. The aurora-related kinase AIR-2 recruits ZEN-4/CeMKLP1 to the mitotic spindle at metaphase and is required for cytokinesis. Curr. Biol. 10, 1162-1171 (2000).

85. Murata-Hori, M. et al. Myosin II regulatory light chain as a novel substrate for AIM-1, an aurora/Ipl1p-related kinas from rat. J . Biochem. (Tokyo) 128, 903-907 (2000).

86. Goto, H. et al. Aurora-B regulates the cleavage furrowspecific vimentin phosphorylation in the cytokinetic process. J. Biol. Chem. 278, 8526-8530 (2003).

87. Kawajiri, A. et al. Functional significance of the specific sites phosphorylated in desmin at cleavage furrow: Aurora-B may phosphorylate and regulate Type III intermediate filaments during cytokinesis coordinatedly with Rho-kinase. Mol. Biol. Cell 14, 1489-1500 (2003)

88. J antsch-Plunger, V. et al. CYK-4: A Rho family GTPase activating protein (GAP) required for central spindle formation and cytokinesis. J. Cell Biol. 149, 1391-1404 (2000).

89. Minoshima, Y., et al. Phosphorylation by Aurora B converts MgcRacGAP to a RhoGAP during cytokinesis. Dev. Cell 4, 549-560 (2003)

An elegant study that identifies a new substrate for Aurora B and shows how the kinase might participate in the regulation of cytokinesis.

90. Kishi, K., Sasaki, T., Kuroda, S., Itoh, T. \& Takai, Y. Regulation of cytoplasmic division of Xenopus embryo by rho p21 and its inhibitory GDP/GTP exchange protein (rho GDI). J. Cell Biol. 120, 1187-1195 (1993).

91. Mabuchi, I. et al. A rho-like protein is involved in the organisation of the contractile ring in dividing sand dollar eggs. Zygote 1, 325-331 (1993).
92. Biggins, S. \& Murray, A. W. The budding yeast protein kinase Ipl1/Aurora allows the absence of tension to activate the spindle checkpoint. Genes Dev. 15, 3118-3129 (2001)

93. Carvalho, A., Carmena, M., Sambade, C., Earnshaw, W. C. $\&$ Wheatley, S. P. Survivin is required for stable checkpoint activation in taxol-treated HeLa cells. J. Cell Sci. 116, 2987-2998 (2003).

94. Lens, S. M. A. et al. Survivin is required for a sustained spindle checkpoint arrest in response to lack of tension EMBO J . 22, 2934-2947 (2003).

References 93 and 94 use RNAi technology to show that survivin is required for the spindle-assembly checkpoint in the presence of mic rotubules, and for BubR1 targeting to the kinetochore.

95. Sugiyama, K. et al. Aurora-B associated protein phosphatases as negative regulators of kinase activation. Oncogene 21, 3103-3111 (2002).

96. Wheatley, S. P., Canvalho, A., Vagnarelli, P. \& Earnshaw, W. C. INCENP is required for proper targeting of survivin to the centromeres and the anaphase spindle during mitosis. Curr. Biol. 11, 886-890 (2001).

97. Bolton, M. A. et al. Aurora B kinase exists in a complex with survivin and INCENP and its kinase activity is stimulated by survivin binding and phosphorylation. Mol. Biol. Cell 13, 3064-3077 (2002).

A thorough study of the regulation of Aurora-B kinase activity by INCENP and survivin.

98. Wheatley, S. P., Kandels-Lewis, S. E., Adams, R. R Ainsztein, A. M. \& Earnshaw, W. C. INCENP binds directly to tubulin and requires dynamic microtubules to target to the cleavage furrow. Exp. Cell. Res. 262, 122-127 (2001).

99. Ainsztein, A. M., Kandels-Lewis, S. E., Mackay, A. M. \& Earnshaw, W. C. INCENP centromere and spindle targeting: identification of essential conserved motifs and involvement of heterochromatin protein HP1. J. Cell Biol. 143, 1763-1774 (1998).

100. Terada, Y., Katayama, H., Tatsuka, M. \& Kuriyama, R AIM-1 regulates onset of cytokinesis by targeting INCENP to midzone and midbody. Mol. Biol. Cell 11S, 343a (2000).

101. Bishop, J . D. \& Schumacher, J. M. Phosphorylation of the carboxyl terminus of inner centromere protein (INCENP) by the Aurora B kinase stimulates Aurora B kinase activity. J. Biol. Chem. 277, 27577-27580 (2002). Provided a clear demonstration that INCENP phosphorylation by Aurora $B$ is part of a feedback loop that stimulates kinase activity.

102. Deveraux, Q. L. \& Reed, J. C. IAP family proteins suppressors of apoptosis. Genes Dev. 13, 239-252 (1999).

103. Uren, A. G. et al. Survivin and the inner centromere protein INCENP show similar cell-cycle localization and gene knockout phenotype. Curr. Biol. 10, 1319-1328 (2000)

104. Chen, J . et al. Survivin enhances Aurora-B kinase activity and localizes Aurora-B in human cells. J. Biol. Chem. 278 486-490 (2003).

105. Morishita, J . et al. Bir1/Cut17 moving from chromosome to spindle upon the loss of cohesion is required for condensation, spindle elongation and repair. Genes Cells $\mathbf{6}$, 743-763 (2001).

106. Martineau-Thuillier, S., Andreassen, P. R. \& Margolis, R. L. Colocalization of TD-60 and INCENP throughout G2 and mitosis: evidence for their possible interaction in signalling cytokinesis. Chromosoma (Berl.) 107, 461-470 (1998).

107. Mollinari, C. et al. The mammalian passenger protein TD-60 is an RCC 1 family member with an essential role in prometaphase to metaphase progression. Dev. Cell 5, 295-307 (2003)

108. Ohi, R., Coughlin, M. L., Lane, W. S. \& Mitchison, T. J . An inner centromere protein that stimulates the microtubule depolymerizing activity of a Kinl kinesin. Dev. Cell 5, 309-321 (2003).

109. Romano, A. et al. CSC-1: a subunit of the Aurora B kinase complex that binds to the survivin-like protein $B I R-1$ and the incenp-like protein ICP-1.J. Cell Biol. 161, 229-236 (2003).

110. Tatsuka, M. et al. Multinuclearity and increased ploidy caused by overexpression of the aurora- and Ipl1-like midbody-associated protein mitotic kinase in human cance cells. Cancer Res. 58, 4811-4816 (1998)

111. Takahashi, T. et al. Centrosomal kinases, HsAIRKI and HsAIRK3, are overexpressed in primary colorectal cancers. J pn. J. Cancer Res. 91, 1007-1014 (2000).

112. Zhou, H. et al. Tumour amplified kinase STK15/BTAK induces centrosome amplification, aneuploidy and transformation. Nature Genet. 20, 189-193 (1998).

113. Tanner, M. M. et al. Frequent amplification of chromosomal region 20q12-q13 in ovarian cancer. Clin. Cancer Res. 6, 1833-1839 (2000).

114. Boveri, T. Zur Frage der Entstehung maligner Tumoren (Fischer Verlag, J ena, 1914). 
115. Doxsey, S. Duplicating dangerously: linking centrosome duplication and aneuploidy. Mol. Cell 10, 439-440 (2002)

116. Meraldi, P., Honda, R. \& Nigg, E. A. Aurora-A overexpression reveals tetraploidization as a major route to centrosome amplification in $\mathrm{p} 53^{-1-}$ cells. EMBO J. 21, 483-492 (2002).

Presents data indicating that centrosome amplification in response to Aurora kinase overexpression arises as a result of failures in cytokinesis.

117. Anand, S., Penrhyn-Lowe, S. \& Venkitaraman, A. R. AURORA-A amplification overrides the mitotic spindle assembly checkpoint, inducing resistance to Taxol. Cancer Cell 3, 51-62 (2003).

118. Chen, S. S., Chang, P. C., Cheng, Y. W., Tang, F. M. \& Lin, Y. S Suppression of the STK15 oncogenic activity requires a transactivation-independent p53 function. EMBO I . 21 4491-4499 (2002).

119. Minn, A. J., Boise, L. H. \& Thompson, C. B. Expression of $\mathrm{Bcl}-\mathrm{xL}$ and loss of p53 can cooperate to overcome a cell cycle checkpoint induced by mitotic spindle damage. Genes Dev. 10, 2621-2631 (1996).

120. Lanni, J. S. \& J acks, T. Characterization of the p53dependent postmitotic checkpoint following spindle disruption. Mol. Cell. Biol. 18, 1055-1064 (1998).

121. Khan, S. H. \& Wahl, G. M. p53 and pRb prevent rereplication in response to microtubule inhibitors by mediating a reversible G1 arrest. Cancer Res. 58, 396-401 (1998).
122. Casenghi, M. et al. p53-independent apoptosis and p53dependent block of DNA rereplication following mitotic spindle inhibition in human cells. Exp. Cell Res. 250, 339-350 (1999).

123. Andreassen, P. R Lohez, O. D Lacroix, F. B. \& Margolis, R. L. Tetraploid state induces p53-dependent arrest of nontransformed mammalian cells in G1. Mol. Biol. Cell 12, 1315-1328 (2001)

124. Gigoux, V., L'Hoste, S., Raynaud, F., Camonis, J \& \&arbay, C. Identification of Aurora kinases as RasGAP Src homology 3 domain-binding proteins. I. Biol. Chem. 277, 23742-23746 (2002).

125. Ota, T. et al. Increased mitotic phosphorylation of histone $\mathrm{H} 3$ attributable to AIM-1/Aurora-B overexpression contributes to chromosome number instability. Cancer Res. 62 5168-5177 (2002)

126. Gautier, J ., Norbury, C., Lohka, M., Nurse, P. \& Maller, J. Purified maturation-promoting factor contains the product of a Xenopus homolog of the fission yeast cell cycle control gene cdc2 + Cell 54, 433-439 (1988).

127. Lohka, M., Hayes, M. K. \& Maller, J. L. Purification of maturation-promoting factor, an intracellular regulator of early mitotic events. Proc. Natl Acad. Sci. USA 85 3009-3013 (1988)

128. Pavletich, N. P. Mechanisms of cyclin-dependent kinase regulation: structures of $\mathrm{Cdks}$, their cyclin activators, and Cip and INK4 inhibitors. J. Mol. Biol. 287, 821-828 (1999).

129. Kufer, T. A. et al. Human TPX2 is required for targeting AuroraA kinase to the spindle. J . Cell Biol. 158, 617-623 (2002).
Acknowledgements

The authors want to thank M. O. Lombardia, University of York, for his generous help and advice on the analysis and modelling of the structural similarities of the kinase domains and for his contribution to figures 1 and 2. Work in the W.C.E. laboratory is supported by The Wellcome Trust, of which W.C.E. is a Principal Research Fellow.

\section{(4) Online links}

DATABASES

The following terms in this article are linked online to:

FlyB ase: http://flybase.bio.indiana.edu/

aurora|D-TACC

LocusLink: http://www.ncbi.nlm.nih.gov/LocusLink/

Aurora A | Aurora B | Aurora C

Saccharomyces Genome Database:

http://www.yeastgenome.org/

Bir1 | Cse4 | Dam1 | Ipl1 |

Ndc10 | Sli15

The Sanger Institute, S. pombe

http://www.sanger.ac.uk/Projects/S_pombe/

Ark1 |Cut17 | Rec8

SwissProt: http://us.expasy.org/sprot/

CENP-A |Eg5 | MgcRacGAP | Mos | p53 | Plk1 | survivin |

TD-60 |TPX2

Access to this interactive links box is free online. 